\title{
REPRESENTATIONS OF RELATIVELY COMPLEMENTED MODULAR LATTICES $\left({ }^{1}\right)$
}

\author{
BY \\ BJARNI JONSSON
}

Introduction. A module over a ring will be said to be locally projective if and only if every finitely generated submodule is projective. As will be shown (7.14), it readily follows from known facts that if $M$ is a locally projective module over a regular ring $R$, then the set $L(M, R)$ of all finitely generated submodules of $M$ is a relatively complemented modular lattice.

This paper is concerned with the representation problem suggested by the above observation. The fundamental theorem, 8.2, gives sufficient conditions in order for a relatively complemented modular lattice $B$ to be isomorphic to $L(M, R)$ for some locally projective module $M$ over a regular ring $R$. Essential use will be made of the results in Jónsson [7], and henceforth that paper will be referred to briefly as CM. In particular, the embedding theorem CM3.2 plays a fundamental role in the construction of the representation module. In $\mathrm{CM}$ this result was proved for complemented Arguesian lattices only, but an easy extension to the relatively complemented case is given in $\$ 1$ of the present paper. $\$ \S 2-8$ are devoted to the construction of the representation module, and to the proof of the fundamental representation theorem. The method is roughly comparable to an approach to the classical coordinatization theorem for projective geometries that combines ideas found in Baer [2] and in Artin [1] as follows: First the given space is embedded as a hyperplane in a space of higher dimension. The old space is regarded as the hyperplane at infinity and the new points as the points of an affine space. The translations of this space form an Abelian group $T$, and the trace-preserving endomorphisms of $T$ form a division ring $E$. The group $T$ is regarded as a vector space over $E$, and to each point at infinity there corresponds in an obvious manner a one-dimensional subspace of $T$. This yields the homogeneous coordinates of the points of the original space.

To a remarkable extent this can be imitated here, although there are complications due to the fact that the given lattice has been embedded in a larger one, and it is therefore necessary to single out a subgroup $T_{0}$ of "admis-

Received by the editors June 13, 1961.

(1) These investigations were supported in part by a grant from the National Science Foundation. The principal results contained in this paper were announced at the Symposium on Lattice Theory in Monterey, California, April 16-17, 1959, and they appear, without proofs, in [5]. 
sible" translations and a subring $E_{0}$ of "special" trace-preserving endomorphisms, and to show that $E_{0}$ is a regular ring, $T_{0}$ is a locally projective module over $E_{0}$, and $B \cong L\left(T_{0}, E_{0}\right)$.

In $\$ 9$ additional conditions are imposed on $B$ that insure that the representation module is projective, and this is used to obtain a generalization of the coordinatization theorem CM8.3. By a generalized regular ring is meant a ring, with or without identity, with the property that for every element $a$ there exists an element $x$ such that $a x a=a$. It is known (cf. Fryer and Halperin $[4$, p. 14]) that if $R$ is a generalized regular ring, then the set $L(R)$ of all principal right ideals of $R$ is a relatively complemented modular lattice. A lattice $B$ is said to be coordinatizable if and only if $B \cong L(R)$ for some generalized regular ring $R$. The applications of the representation theorem to the coordinatization problem are based on the observation that if $M$ is a projective module over a regular ring, then $L(M, R)$ is coordinatizable. In fact, the endomorphisms of $M$ whose range is finitely generated form a generalized regular ring $R^{\prime}$ with $L(M, R) \cong L\left(R^{\prime}\right)$.

$\$ 10$ contains some further results on coordinatizable lattices, and an example given there shows that the natural extension of the isomorphism theorem CM9.4 to the relatively complemented case does not hold. It should also be observed that by the methods of this section the main results of $\$ 9$ can be easily obtained from the coordinatization theorem CM8.3, without the use of the Representation Theorem 8.2.

All the lattices considered here will be assumed to have a zero element 0 . The symbols + and $\cdot$ will be used for the lattice operations of addition and multiplication, and $\leqq$ for the inclusion relation. Even when the lattice involved consists of subgroups of a group, and the operation of multiplication and the relation of inclusion coincide with set-theoretic intersection and inclusion, these symbols will usually be used in preference to the standard settheoretic notation. Perspectivity will be denoted by $\sim$, and if the elements $a_{0}, a_{1}, \cdots, a_{n-1}$ are independent, this will be indicated by writing $\left(a_{0}, a_{1}, \cdots, a_{n-1}\right) \perp$. The fact that the terms of a sum are independent will also be indicated by placing a dot over the plus sign:

$$
a_{0}+a_{1}+\cdots+a_{n-1} \text {. }
$$

If $B$ is a sublattice of a lattice $A$, and if $a \in A$, then $B a$ is the complex product consisting of all elements $x a$ with $x \in B$. Of course, if $a \in B$, then $B a$ is simply the principal ideal of $B$ that is generated by $a$, but if $a \notin B$, then $B a$ need not be a subset of $B$. However, in the situations in which this notation is used here $B a$ is actually always a subset of $B$, and hence an ideal although not necessarily a principal ideal.

The notion of a partial $n$-frame of a lattice with a unit element $u$ was introduced in CM. This is a system $a$ of elements $a_{i}, a_{i, j}, a^{*}, i, j=0,1, \cdots, n-1$, such that $u=a_{0} \dot{+} a_{1} \dot{+} \cdots+a_{n-1} \dot{+} a^{*}, a_{i}+a_{j}=a_{i} \dot{+} a_{i, j}, a_{i, j}=a_{j, i}$ and $a_{i, j}$ 
$=\left(a_{i, p}+a_{p, j}\right)\left(a_{i}+a_{j}\right)$ for $i, j, p=0,1, \cdots, n-1$. An $n$-frame $a$ is said to be large in case $a^{*}$ is the sum of finitely many elements, each of which is perspective to a part of $a_{0}$. In the particular case when $a^{*}=0$ the partial $n$-frame reduces to the older concept of a homogeneous $n$-frame introduced by von Neumann.

If the lattice under consideration does not have a unit element, then the concept of a partial $n$-frame cannot be applied to the whole lattice, but only to certain principal ideals. In this connection another notion is needed: An element $b$ of a modular lattice $B$ is said to be large if and only if every member of $B$ is the sum of a finite number of elements each of which is perspective to a part of $b$. The following remark gives some hint concerning the intended use of these concepts: The fundamental assumption concerning the complemented modular lattices studied in CM was that they possess a large partial $n$-frame. Here the corresponding restriction imposed on the relatively complemented modular lattices $B$ will be that there exist a large element $d$ such that the principal ideal $B d$ possesses a homogeneous $n$-frame.

1. The embedding theorem. In this section the principal embedding theorem of $\mathrm{CM}$ will be extended to relatively complemented Arguesian lattices. For this purpose it must be observed that the method given in Frink [3] for embedding a complemented modular lattice in a projective geometry applies equally well to relatively complemented lattices, and that the theorem of Jónsson [6] which asserts that this embedding preserves all identities is also valid in the more general setting. Fortunately the arguments given in these papers carry over essentially unchanged, and do not have to be repeated.

THEOREM 1.1. Every relatively complemented Arguesian lattice is isomorphic to a sublattice of the lattice of all subgroups of an Abelian group.

Outline of proof. As in the complemented case (Frink [3]), the maximal proper dual ideals of the given lattice $B$ are the points of a projective geometry, the line through two distinct points $M$ and $N$ being the set of all points $P$ such that $M \cap N \subseteq P$. The mapping that associates with each member $a$ of $B$ the set of all points $M$ containing $a$ is an isomorphism of $B$ onto a sublattice $B$ of the lattice $a$ of all subspaces of this geometry.

The definition in [6] of a regular sublattice of a projective lattice can be modified by not requiring the sublattice to contain a unit element, and it follows as there that $B$ is a regular sublattice of $a$. Thus every relatively complemented modular lattice $B$ is a regular sublattice of a projective lattice $A$.

Again, the same argument as in [6] shows that even for relatively complemented lattices this embedding preserves all identities that hold in $B$. In particular, if $B$ is Arguesian, then so is $A$. In this case, therefore, each irreducible direct factor (summand) of $A$ is Arguesian, and is therefore either isomorphic to the lattice of all subspaces of a vector space over a division 
ring, or else is of dimension 1 or 2. Therefore each direct factor of $A$ is isomorphic to a sublattice of the lattice of all subgroups of an Abelian group, and the same is therefore true of $A$ and of $B$.

THEOREM 1.2. If $B$ is a relatively complemented sublattice of the lattice of all subgroups of an Abelian group $v$, and if $b$ is a homogeneous 3-frame of a principal ideal of $B$, then there exist an Abelian group $u^{\prime}$ containing $v$ as a subgroup, a relatively complemented sublattice $B^{\prime}$ of the lattice $A^{\prime}$ of all subgroups of $u^{\prime}$, and a partial 3-frame $a$ of $A^{\prime}$ such that $a^{*}=v, a_{i}, a_{i, j} \in B^{\prime}$ for $i, j=0,1,2$, $a_{i} \sim b_{0}$ in $B^{\prime}$ for $i=0,1,2$, and $B^{\prime}=B^{\prime} a_{0}+B^{\prime} a_{1}+B^{\prime} a_{2}+B$.

Proof. Let $u^{\prime}$ be the direct sum of $v$ and of three groups $c_{0}, c_{1}, c_{2}$ that are isomorphic to $b_{0}$. In the lattice $A^{\prime}$ of all subgroups of $u^{\prime}$, each of the groups $c_{i}$ is therefore perspective to $b_{0}$ with an axis of perspectivity $d_{i}$. Consequently a partial 3-frame $a$ of $A^{\prime}$ can be defined by letting $a_{i}=c_{i}, a_{i, j}=\left(a_{i}+a_{j}\right)\left(d_{i}+d_{j}\right)$ and $a^{*}=v$. Let $B^{\prime}$ be the sublattice of $A^{\prime}$ generated by $B$ together with the elements $a_{i}, d_{i}$. It will be shown by three successive applications of CM3.2 that $B^{\prime}$ has the desired properties.

Let $D$ be the dual ideal of $B$ that is generated by $b_{0}+b_{1}+b_{2}$,

$$
D=\left\{x \mid b_{0}+b_{1}+b_{2} \leqq x \in B\right\},
$$

and for $x \in D$ let $x^{\prime}=a_{0}+x$,

$$
F(x)=\left\{y \mid x^{\prime} \geqq y \in A^{\prime}\right\}, \quad G(x)=\{y \mid x \geqq y \in B\},
$$

and let $H(x)$ be the sublattice of $F(x)$ generated by the set

$$
G(x) \cup\left\{a_{0}, d_{0}\right\} .
$$

Given $x \in D$, there exists a 4-frame $a^{\prime}$ of $F(x)$ such that $a_{0}^{\prime}=a_{0}, a_{i}^{\prime}=b_{i-1}$ for $i=1,2,3, a_{0,1}^{\prime}=d_{0}, a_{i, j}^{\prime}=b_{i-1, j-1}$ for $i, j=1,2,3$, and $a^{\prime *} \in G(x)$. Consequently, by CM3.2, $H(x)$ is complemented and $H(x)=H(x) a_{0}+G(x)$. Since the lattices $G(x)$ form a directed system whose union is $B$, and the lattices $H(x)$ form a directed system whose union is the lattice $B_{0}$ generated by $B \cup\left\{a_{0}, d_{0}\right\}$, this implies that

$$
B_{0}=B_{0} a_{0}+B .
$$

By a second application of CM3.2, with $B$ replaced by $B_{0}$, it is found that the set $B_{0} \cup\left\{a_{1}, d_{1}\right\}$ generates a relatively complemented lattice $B_{1}$ with

$$
B_{1}=B_{1} a_{1}+B_{0},
$$

and a third application of CM3.2 shows that the set $B_{1} \cup\left\{a_{2}, d_{2}\right\}$ generates a relatively complemented lattice $B_{2}$ with

$$
B_{2}=B_{2} a_{2}+B_{1} \text {. }
$$

The proof is now completed by observing that $B^{\prime}=B_{2}$. 
2. Translations. In the proof of the principal theorem of this paper, the Representation Theorem 8.2, it will be assumed that the given lattice has been embedded in the lattice of all subgroups of an Abelian group $v$, in accordance with 1.1 , and that $v$ has been embedded in a larger group $u^{\prime}$ as in 1.2 , and the whole structure described in 1.2 will be involved in the construction of the representation module. This section and the next one, however, involve only an Abelian group $u^{\prime}$ and a direct summand $u$ which later will be identified with the group $a_{1}+a_{2}+v$ of 1.2. A class $T$ of automorphisms of $u^{\prime}$ will be defined, whose members are called translations, and it will be shown that under these automorphisms the complements of $u$ in $u^{\prime}$ behave somewhat like points of an affine geometry. Thus $T$ is an Abelian group and for any two "points" $x$ and $y$ there is a unique translation that maps $x$ onto $y$. Also, it is possible to associate with each member $f$ of $T$ its trace, or direction, which is represented by a certain subgroup $\pi(f)$ of $u$, and in terms of this concept the notion of a trace-preserving endomorphism can be defined.

The Abelian group $u^{\prime}$ and the direct summand $u$ will be assumed to be fixed throughout this section.

Definition 2.1. By a point will be meant a complement of $u$ in $u^{\prime}$. By a translation will be meant an automorphism $f$ of $u^{\prime}$ such that $\alpha-f(\alpha) \in u$ for all $\alpha \in u^{\prime}$ and $f(\alpha)=\alpha$ for all $\alpha \in u$. The set of all translations will be called $T$.

There is a close connection between translations on one hand and projections whose kernel is $u$ on the other. Since this connection can be utilized to an advantage here, and will play an important role in some of the later work, it is convenient to have a name for the class of these projections.

Definition 2.2. $P$ is the class of all projections of $u^{\prime}$ whose kernel is $u$.

Thus an endomorphism $p$ of $u^{\prime}$ belongs to $P$ if and only if $p p=p, p\left(u^{\prime}\right)$ is a point, and $p(u)=0$.

Corollary 2.3. Suppose $p \in P$ and $q$ is an endomorphism of $u^{\prime}$. Then $q \in P$ if and only if $p q=p$ and $q p=q$.

Proof. If $q \in P$, then for all $\alpha \in u^{\prime}$,

$$
\begin{aligned}
& q q(\alpha)=q(\alpha), \quad q(\alpha-q(\alpha))=0, \quad \alpha-q(\alpha) \in u, \\
& p(\alpha-q(\alpha))=0, \quad p q(\alpha)=p(\alpha) .
\end{aligned}
$$

Thus $p q=p$ and, similarly, $q p=q$. Conversely, the conditions $p q=p$ and $q p=q$ jointly imply that $p$ and $q$ have the same kernel and that $q q=q p q=q p=q$, hence that $q \in P$.

Corollary 2.4. If $p, q \in P$ and $f=1-p+q$, then $f \in T$ and $f^{-1}=1-q+p$. Conversely, if $p \in P, f \in T$ and $q=f p$, then $q \in P$ and $f=1-p+q$.

Proof. Under the hypothesis of the first part, if $g=1-q+p$, then $f g=g f=1$ by 2.3 , so that $f$ is an automorphism of $u^{\prime}$ and $g=f^{-1}$. Also, $p(1-f)=p(p-q)$ 
$=0$, so that $1-f$ maps $u^{\prime}$ into $u$. Finally, $f(1-p)=f-f p=f-q=1-p$, whence it follows that $f(\alpha)=\alpha$ for all $\alpha \in u$.

Under the hypothesis of the second part, $1-f$ maps $u^{\prime}$ onto $u$, whence it follows that $p(1-f)=0, p f=p, p q=p f p=p p=p$. Since also, $q p=f p p=f p=q$, it follows by 2.3 that $q \in P$. Furthermore, $1-p$ maps $u^{\prime}$ into $u$ because $p(1-p)=p-p p=0$. Therefore $f(1-p)=1-p, f-q=1-p, f=1-p+q$.

Corollary 2.5. If $p, q, r \in P, f=1-p+q$ and $g=1-q+r$, then $f g=g f$ $=1-p+r$.

Proof. By 2.3 and 2.4.

THEOREM 2.6. $T$ is an Abelian group under superposition.

Proof. By 2.4 and 2.5 .

Corollary 2.7. Suppose $p \in P$ and $f$ is an endomorphism of $u^{\prime}$. Then $f \in T$ if and only if $p f=p$ and $f p=f-1+p$.

Proof. The forward implication follows from the second part of 2.4. Conversely, assuming that $p f=p$ and $f p=f-1+p$, let $q=f p$. Then $p q=p f p=p p$ $=p$ and $q p=f p p=f p=q$, so that $q \in P$. Since $f=1-p+q$, it follows by the first part of 2.4 that $f \in T$.

Corollary 2.8. For any points $b$ and $c$ there exists a unique translation $f$ such that $f(b)=c$.

Proof. If $p, q$ are the members of $P$ that map $u^{\prime}$ onto $b$ and $c$, respectively, then by 2.4, $f=1-p+q$ is a translation with $f p=q$. Therefore $f(b)=f p\left(u^{\prime}\right)$ $=q\left(u^{\prime}\right)=c$.

Given $f \in T$ with $f(b)=c$, let $p$ be the member of $P$ that maps $u^{\prime}$ onto $b$, and let $q=f p$. Then by 2.4, $q \in P$ and $f=1-p+q$. Also, $q\left(u^{\prime}\right)=f p\left(u^{\prime}\right)=f(b)$ $=c$. Thus $q$ is the unique member of $P$ that maps $u^{\prime}$ onto $c$. This proves the uniqueness of $f$.

CoROllary 2.9. If $f$ is a translation, and if the condition $f(x)=x$ holds for one point $x$, then $f=1$.

Proof. Since the identity automorphism is a translation, this follows from the uniqueness part of 2.8 .

COROLlARY 2.10. If $f$ is a translation, and if the condition $x f(x)=0$ holds for one point $x$, then this condition holds for every point $x$.

Proof. Consider two points $x$ and $y$. By 2.8 there exists a translation $g$ such that $g(x)=y$. Hence, by 2.6 ,

$$
y f(y)=g(x) f g(x)=g(x) g f(x)=g(x f(x)),
$$

whence it follows that $y f(y)=0$ if and only if $x f(x)=0$. 
LEMMA 2.11. If $f \in T, b$ is a point, and $c=f(b)$, then for any subgroup $x$ of $u^{\prime}$, the condition $(b+c) x \leqq b$ implies that

$$
f(x)=[(b+c) u+x][(b+x) u+c] .
$$

Proof. If $\alpha \in x$, then there exists $\beta$ such that $\beta \in b$ and $\alpha-\beta \in u$. Therefore $(\beta) \in c$ and $\beta-f(\beta) \in u$, so that $\beta-f(\beta) \in(b+c) u$. Also $f(\alpha)-f(\beta)=f(\alpha-\beta)$ $=\alpha-\beta$, so that $\alpha-f(\alpha)=\beta-f(\beta)$. Consequently

$$
\begin{aligned}
& f(\alpha)=(f(\alpha)-\alpha)+\alpha \in(b+c) u+x, \\
& f(\alpha)=(\alpha-\beta)+f(\beta) \in(b+x) u+c .
\end{aligned}
$$

Thus

$$
f(x) \leqq[(b+c) u+x][(b+x) u+c]
$$

Now suppose

$$
\xi \in[(b+c) u+x][(b+x) u+c] .
$$

Then there exist $\alpha, \beta$ such that

$$
\alpha \in x, \quad \xi-\alpha \in(b+c) u, \quad \beta \in c, \quad \xi-\beta \in(b+x) u,
$$

and consequently there exist $\gamma, \delta$ such that

$$
\gamma \in b, \quad \alpha-\gamma \in u \quad \delta \in x, \quad \xi-\beta-\delta \in b .
$$

It follows that

$$
\alpha-\delta=(\alpha-\xi)+(\xi-\beta-\delta)+\beta \in b+c \text { and } \alpha-\delta \in x,
$$

so that, by the hypothesis, $\alpha-\delta \in b$. Consequently

$$
\xi-\beta-\alpha+\gamma=(\xi-\beta-\delta)-(\alpha-\delta)+\gamma \in b .
$$

But also,

$$
\xi-\beta-\alpha+\gamma=(\xi-\beta)-(\alpha-\gamma) \in u,
$$

and since $b u=0$, this implies that

$$
\xi-\beta-\alpha+\gamma=0, \quad \xi=\alpha+\beta-\gamma .
$$

From the fact that $\beta-\xi, \xi-\alpha$ and $\alpha-\gamma$ belong to $u$ it follows that $\beta-\gamma \in u$. Inasmuch as $\gamma \in b$ and $\beta \in c=f(b)$, this implies that $\beta=f(\gamma)$. Therefore

$$
f(\alpha)=f(\gamma)+f(\alpha-\gamma)=\beta+(\alpha-\gamma)=\xi .
$$

Thus $\xi \in f(x)$.

The inequality (1) can therefore be replaced by an identity, and the proof is complete.

Lemma 2.12. If $f \in T$, then for all points $b, c$,

$$
[b+f(b)] u=[c+f(c)] u .
$$


Proof. If $\xi \in[b+f(b)] u$, then for some $\alpha$,

$$
\alpha \in b, \quad \xi-\alpha \in f(b),
$$

and hence for some $\beta$,

$$
\beta \in c, \quad \alpha-\beta \in u \text {. }
$$

It follows that

$$
f(\alpha)=\alpha-\xi \text { and } f(\alpha)-f(\beta)=f(\alpha-\beta)=\alpha-\beta .
$$

Consequently

$$
\xi=\alpha-f(\alpha)=\beta-f(\beta) \in[c+f(c)] u .
$$

Thus

$$
[b+f(b)] u \leqq[c+f(c)] u .
$$

Definition 2.13. By the trace of a translation $f$, in symbols $\pi(f)$, will be meant the unique subgroup $d$ of $u$ such that, for all points $b$,

$$
d=[b+f(b)] u \text {. }
$$

Corollary 2.14. For all $f, g \in T, \pi(f g) \leqq \pi(f)+\pi(g)$.

Proof. If $b$ is a point, then so is $f(b)$, and

$$
\begin{aligned}
\pi(f)+\pi(g) & =[b+f(b)] u+[f(b)+g f(b)] u \\
& =[[b+f(b)] u+f(b)+g f(b)] u \\
& =[[b+f(b)][u+f(b)]+g f(b)] u \\
& =[b+f(b)+g f(b)] u \\
& \geqq[b+g f(b)] u=\pi(f g) .
\end{aligned}
$$

Corollary 2.15. For all $f \in T, \pi(f)=\pi\left(f^{-1}\right)$.

Proof. If $b$ is a point, then so is $c=f(b)$, and

$$
\pi\left(f^{-1}\right)=\left[c+f^{-1}(c)\right] u=[f(b)+b] u=\pi(f) .
$$

3. Trace-preserving endomorphisms. This section, like the preceding one, will be concerned with a fixed Abelian group $u^{\prime}$ and a direct summand $u$ of $u^{\prime}$. Since in the Abelian group $T$ the group operation (superposition) is written multiplicatively, it is convenient when speaking of endomorphisms of $T$ to use exponential notation. Thus $f^{*}$ is the image of the translation $f$ under the endomorphism $s$ of $T$, and if $s$ and $t$ are endomorphisms of $T$, then $s+t, s t$ and $-s$ are the endomorphisms of $T$ such that, for all $f \in T$,

$$
f^{s+t}=f^{s} f^{t}, \quad f^{s t}=\left(f^{s}\right)^{t}, \quad f^{-s}=\left(f^{s}\right)^{-1} \text {. }
$$

The symbol 1 will be used to denote the identity endomorphism of $T\left(f^{1}=f\right)$ 
as well as the identity translation, and the zero element of the ring of endomorphisms will be denoted by the symbol $0\left(f^{0}=1\right)$.

DeFINITION 3.1. An endomorphism sof $T$ will be said to be trace-preserving if and only if $\pi\left(f^{a}\right) \leqq \pi(f)$ for every translation $f$. The set of all trace-preserving endomorphisms of $T$ will be called $E$.

Lemma 3.2. If $b$ is a point, then for all translations $f$ and $g$ the conditions $\pi(f) \leqq \pi(g)$ and $f(b) \leqq b+g(b)$ are equivalent.

Proof. Obvious.

CoROllary 3.3. $E$ is a subring of the ring of all endomorphisms of $T$, containing the identity endomorphism of $T$.

Proof. Clearly $1 \in E$. Also, if $s, t \in E$, then for all $f \in T$,

$$
\begin{aligned}
\pi\left(f^{s+t}\right) & =\pi\left(f^{s} f^{t}\right) \leqq \pi\left(f^{s}\right)+\pi\left(f^{t}\right) \leqq \pi(f), \\
\pi\left(f^{s}\right) & =\pi\left(\left(f^{s}\right)^{t}\right) \leqq \pi\left(f^{s}\right) \leqq \pi(f), \\
\pi\left(f^{s}\right) & =\pi\left(f^{s}\right) \leqq \pi(f),
\end{aligned}
$$

so that $s+t, s t,-s$ belong to $E$.

In an affine geometry that satisfies Desargues' Law, if two translations $h$ and $h^{\prime}$ are such that $\pi\left(h^{\prime}\right) \leqq \pi(h)$, then there exists a unique trace-preserving endomorphism $s$ such that $h^{\prime}=h^{\text {s }}$. It will now be shown that, in the present situation, if $b h(b)=0$ for one point $b$, and hence for every point $b$, and if $\pi\left(h^{\prime}\right) \leqq \pi(h)$, then $h^{\prime}=h^{s}$ for some $s \in E$. The formula for $s$ is suggested by picturing the points as points on an affine plane and $u$ as the line at infinity. Let $c=h(b)$ and $x=h^{\prime}(b)$ and, assuming that $f$ is a given translation, let $d=f(b)$. If $b, c, d$, are not collinear, then $f^{*}(b)$ should be the point in which the line $b+d$ meets the line through $x$ parallel to $c+d$, i.e., the point

$$
y=(b+d)[(c+d) u+x] .
$$

Let $p_{0}, p, q, r$ be the members of $P$ that map $u^{\prime}$ onto $b, x, d$ and $y$, respectively, and let $p_{1}$ be the projection of $b+c$ onto $c$ with kernel $b$. Then, for every $\alpha \in u^{\prime}$,

$$
\begin{gathered}
p(\alpha) \in x, \quad p_{1} p(\alpha) \in c, \quad p(\alpha)-p_{1} p(\alpha) \in b, \\
q p_{1} p(\alpha) \in d, \quad p_{1} p(\alpha)-q p_{1} p(\alpha) \in u .
\end{gathered}
$$

Hence $p_{1} p(\alpha)-q p_{1} p(\alpha) \in(c+d) u$, and the element

$$
\beta=p(\alpha)-p_{1} p(\alpha)+q p_{1} p(\alpha)
$$

belongs to both $b+d$ and $(c+d) u+x$, and therefore belongs to $y$. Since the element

$$
\alpha-\beta=(\alpha-p(\alpha))+\left(p_{1} p(\alpha)-q p_{1} p(\alpha)\right)
$$

belongs to $u$, it follows that $r(\alpha)=\beta$. Thus 


$$
r=p-p_{1} p+q p_{1} p=p+\left(f-2+p_{0}\right) p_{1} p .
$$

From the fact that $p-p_{1} p$ maps $u^{\prime}$ into $b$ it follows that

$$
p_{0}\left(p-p_{1} p\right)=p-p_{1} p, \quad p_{0}-p_{0} p_{1} p=p-p_{1} p, \quad p_{0} p_{1} p=p_{0}-p+p_{1} p .
$$

Consequently

$$
r=p_{0}+(f-1) p_{1} p
$$

These considerations suggest that the endomorphism $s$ should be defined by the formula

$$
f^{s}=1+(f-1) p_{1} p
$$

It will now be shown that this formula does indeed define an endomorphism with the desired properties.

THEOREM 3.4. Suppose $h, h^{\prime}$ are translations with $\pi\left(h^{\prime}\right) \leqq \pi(h), b$ is a point, and $b h(b)=0$. Let $p$ be the member of $P$ that maps $u^{\prime}$ onto $h^{\prime}(b)$, let $p_{1}$ be the projection of $b+h(b)$ onto $h(b)$ with kernel $b$, and let $s$ be the function on $T$ such that

$$
f^{*}=1+(f-1) p_{1} p \quad \text { for all } f \in T .
$$

Then $s \in E$ and $h^{s}=h^{\prime}$.

Proof. For any translation $f, p f=p$ by 2.7. Therefore $p f^{\bullet}=p$. Also,

$$
f^{\circ} p=p+(f-1) p_{1} p=f^{\circ}-1+p .
$$

Therefore $f^{\circ} \in T$ by 2.7 .

If $f, g \in T$, then there exist $q_{0}, q_{1}, q_{2} \in P$ such that

$$
f=1-q_{0}+q_{1}, \quad g=1-q_{1}+q_{2},
$$

and therefore by 2.5

$$
f g=1-q_{0}+q_{2}
$$

Consequently

$$
\begin{aligned}
f^{8} g^{s} & =\left(1+\left(q_{1}-q_{0}\right) p_{1} p\right)\left(1+\left(q_{2}-q_{1}\right) p_{1} p\right) \\
& =1+\left(q_{2}-q_{0}\right) p_{1} p=(f g)^{8} .
\end{aligned}
$$

The second equality above follows from the fact that $p\left(q_{2}-q_{1}\right)=p-p=0$. Thus $s$ is an endomorphism of $T$.

To show that $\pi\left(f^{s}\right) \leqq \pi(f)$, observe that

$$
f^{s}=p-p_{1} p+f\left(1-p+p_{1} p\right),
$$

because $f(1-p)=1-p$. Now $p-p_{1} p$ maps $b$ into itself, and therefore so does $1-p+p_{1} p$. Consequently $f^{*}(b) \leqq b+f(b)$, and the desired inclusion follows from 3.2. 
Finally, $h=1-p_{0}+q$ where $p_{0}$ and $q$ are the members of $P$ that map $u^{\prime}$ onto $b$ and $h(b)$, respectively. Observe that $q p_{1} p=p_{1} p$ because $p_{1} p$ maps $u^{\prime}$ onto $h(b)$. Also, as was observed in the preliminary discussion, $p_{0} p_{1} p=p_{0}$ $-p+p_{1} p$. Therefore

$$
h^{s}=1+\left(q-p_{0}\right) p_{1} p=1-p_{0}+p=h^{\prime} .
$$

4. Admissible translations. In this section, and in the next three sections, the entire structure arising from the Embedding Theorem 1.2 will be involved. More precisely, the following assumptions will be made.

TEMPORARY ASSUMPTIONS 4.1. Throughout \$\$4-7 it is assumed that

(i) $A^{\prime}$ is the lattice of all subgroups of an Abelian group $u^{\prime}$.

(ii) $A$ is the lattice of all subgroups of a subgroup $u$ of $u^{\prime}$.

(iii) $a$ is a partial 3-frame of $A^{\prime}$ with $\bar{a}_{0}=u$.

(iv) $B^{\prime}$ is a relatively complemented sublattice of $A^{\prime}$ such that $a_{0}, a_{1}, a_{2}, a_{0,1}$, $a_{0,2} \in B^{\prime}$ and $B^{\prime}=B^{\prime} a_{0}+B^{\prime} a_{1}+B^{\prime} a_{2}+B^{\prime} a^{*}$.

It follows that $u$ is a direct summand of $u^{\prime}$; in fact, $u^{\prime}=a_{0}+u$. The group $T$ of translations, the ring $E$ of trace-preserving endomorphisms of $T$, and the set $P$ of projections with kernel $u$ can therefore be defined as in $\S \S 2$ and 3 , and all the results obtained in those sections apply to the present situation.

Definition 4.2. A translation $f$ is said to be admissible if and only if $f\left(B^{\prime}\right)$ $\subseteq B^{\prime}$. The set of all admissible translations will be called $T_{0}$.

Lemma 4.3. If $x, y \in B^{\prime}$, then $x\left(y+a^{*}\right) \in B^{\prime}$ and $x(y+u) \in B^{\prime}$.

Proof. By 4.1 (iv) there exists $z \in B^{\prime} a^{*}$ such that

$$
x+y \leqq a_{0}+a_{1}+a_{2}+z .
$$

Therefore

$$
\begin{aligned}
x\left(y+a^{*}\right) & =x\left(a_{0}+a_{1}+a_{2}+z\right)\left(y+a^{*}\right)=x\left[y+\left(a_{0}+a_{1}+a_{2}+z\right) a^{*}\right] \\
& =x\left[y+z+\left(a_{0}+a_{1}+a_{2}\right) a^{*}\right]=x(y+z) \in B^{\prime} .
\end{aligned}
$$

The second part of the conclusion follows by replacing $y$ by $y+a_{0}+a_{1}+a_{2}$.

THEOREM 4.4. A translation $f$ is admissible if and only if there exists a point $x$ such that $x \in B^{\prime}$ and $f(x) \in B^{\prime}$.

Proof. If $f$ is admissible, then for every point $x, x \in B^{\prime}$ implies that $f(x) \in B^{\prime}$. Conversely, suppose there exists a point $x$ such that both $x$ and $y=f(x)$ belong to $B^{\prime}$. It will be shown that, for every subgroup $z$ of $u^{\prime}$, the condition $z \in B^{\prime}$ implies that $f(z) \in B^{\prime}$. The proof will be divided into four cases.

Case 1. $(x+y) z \leqq x$.

By Lemma 2.11,

$$
f(z)=[(x+y) u+z][(x+z) u+y],
$$


and the conclusion follows with the aid of 4.3.

Case 2. $y \leqq x+a_{2}+a^{*}$ and $z \leqq x+y$.

By CM1.3 there exists $v \in B^{\prime}$ such that

$$
x+a_{1}=x+v=a_{1}+v \text {. }
$$

Then $v$ is a point. Let $w=f(v)$. Since

$$
(x+y) v \leqq\left(x+a_{2}+a^{*}\right)\left(x+a_{1}\right) v=x v=0,
$$

it follows by Case 1 that $w \in B^{\prime}$. Also, by Lemma 2.11,

$$
w \leqq(x+y) u+v \leqq\left(x+a_{2}+a^{*}\right) u+v=a_{2}+a^{*}+v .
$$

Consequently,

$$
(x+y)(v+w) \leqq\left(x+a_{2}+a^{*}\right)\left(a_{2}+a^{*}+v\right)=a_{2}+a^{*} .
$$

The last equality follows from the fact that $\left(x, v, a_{2}, a^{*}\right) \perp$. Since $z u \in B^{\prime}$ by Lemma 4.3, there exists $z^{\prime} \in B^{\prime}$ such that $z=z u+z^{\prime}$. Then

$$
(v+w) z^{\prime}=(v+w)(x+y) z^{\prime} \leqq\left(a_{2}+a^{*}\right) z^{\prime}=0,
$$

so that $f\left(z^{\prime}\right) \in B^{\prime}$ by Case 1 . Inasmuch as $f(z u)=z u \in B^{\prime}$, this implies that $f(z)=f\left(z^{\prime}\right)+f(z u) \in B^{\prime}$.

Case 3. $y \leqq x+a_{2}+a^{*}, z$ arbitrary.

There exists $z^{\prime} \in B^{\prime}$ such that $z=(x+y) z+z^{\prime}$, and it follows by Cases 2 and 1 that $f(z)=f((x+y) z)+f\left(z^{\prime}\right) \in B^{\prime}$.

Case 4. $y$ and $z$ arbitrary.

Let $x^{\prime}=\left(x+a_{1}\right)\left(y+a_{2}+a^{*}\right)$. By 4.3, $x^{\prime} \in B^{\prime}$. Also

$$
\begin{aligned}
x^{\prime}+u & =\left(x+a_{1}\right)\left(y+a_{2}+a^{*}\right)+a_{1}+a_{2}+a^{*} \\
& =\left(x+a_{1}+a_{2}+a^{*}\right)\left(y+a_{1}+a_{2}+a^{*}\right) \\
& =(x+u)(y+u)=u^{\prime}, \\
x^{\prime} u & =\left(x+a_{1}\right) u\left(y+a_{2}+a^{*}\right) u=a_{1}\left(a_{2}+a^{*}\right)=0,
\end{aligned}
$$

so that $x^{\prime}$ is a point.

Let $g$ and $h$ be the translations such that $g(x)=x^{\prime}$ and $h\left(x^{\prime}\right)=y$. Then $f=h g$. Since $x^{\prime} \leqq x+a_{1}$, Case 3 with the subscripts 1 and 2 interchanged implies that $g(z) \in B^{\prime}$, and since

$$
x^{\prime}+a_{2}+a^{*}=\left(x+a_{1}+a_{2}+a^{*}\right)\left(y+a_{2}+a^{*}\right)=y+a_{2}+a^{*} \geqq y,
$$

a second application of Case 3 yields the desired conclusion,

$$
f(z)=h g(z) \in B^{\prime} \text {. }
$$

THEOREM 4.5. $T_{0}$ is a subgroup of $T$.

Proof. Clearly $f g \in T_{0}$ whenever $f, g \in T_{0}$. If $f \in T_{0}$, then $x=f\left(a_{0}\right)$ is a point that belongs to $B^{\prime}$, and $f^{-1}(x)=a_{0}$. Therefore $f^{-1} \in T_{0}$ by 4.4. Finally, $T_{0}$ is 
nonempty because the identity translation belongs to $T_{0}$.

5. Special endomorphisms. In 3.4 a method was given for constructing, for certain translations $h$ and $h^{\prime}$, a trace-preserving endomorphism that maps $h$ onto $h^{\prime}$. This result will now be applied to the special case in which $h$ is the particular translation that maps $a_{0}$ onto $a_{0,1}$, and $h^{\prime}$ is an admissible translation with $\pi\left(h^{\prime}\right) \leqq a_{0}+a_{1}$. The corresponding endomorphisms will be called special endomorphisms. It seems likely that the special endomorphism $s$ could alternatively be characterized by the condition $T_{0}^{8} \subseteq T_{0}$, but no attempt has been made to prove this conjecture, since it seems to be more convenient to work with the explicit formula in 3.4.

Definition 5.1. By $p_{0}$ will be meant the member of $P$ that maps $u^{\prime}$ onto $a_{0}$, and by $p_{1}$ will be meant the projection of $a_{0}+a_{1}$ onto $a_{0,1}$ with kernel $a_{0}$.

DEFINITION 5.2. By a special projection will be meant a member $p$ of $P$ such that $p\left(u^{\prime}\right) \in B^{\prime}$ and $p\left(u^{\prime}\right) \leqq a_{0}+a_{1}$. The set of all special projections will be called $P_{0}$.

DEFINITION 5.3. By the endomorphism associated with a special projection $p$ will be meant the member $s$ of $E$ such that $f^{s}=1+(f-1) p_{1} p$ for all $f \in T . B y a$ special endomorphism will be meant a member $s$ of $E$ that is associated with some special projection. The set of all special endomorphisms will be called $E_{0}$.

Definition 5.4. For $p \in P_{0}$ and $f \in T, p \odot f=1+(f-1) p_{1} p$.

Lemma 5.5. Suppose $p \in P_{0}, f \in T$, and let $q=f-1+p_{0}, r=(p \odot f)-1+p_{0}$. Then $q, r \in P$ and $r=p-p_{1} p+q p_{1} p$.

Proof. By 2.4, $q$ and $r$ belong to $P$. By 5.4,

$$
r=p_{0}+\left(q-p_{0}\right) p_{1} p
$$

As was observed in the last part of the discussion preceding Theorem 3.4, $p_{0} p_{1} p=p_{0}-p+p_{1} p$. The desired formula for $r$ follows by a simple substitution.

Lemma 5.6. For any $p \in P_{0}$ and $f \in T,(p \odot f)\left(a_{0}\right)$ is the set of all $\xi \in u^{\prime}$ such that, for some $\alpha, \beta$,

(i) $\alpha \in a_{0}, \quad \xi-\alpha \in f\left(a_{0}\right), \quad \beta \in p\left(u^{\prime}\right), \quad \xi-\beta \in u, \quad \alpha-\beta \in a_{0,1}$.

Proof. Let $q$ and $r$ be as in 5.5. Then $f\left(a_{0}\right)=q\left(u^{\prime}\right)$ and $(p \odot f)\left(a_{0}\right)=r\left(u^{\prime}\right)$ $=r p\left(u^{\prime}\right)$. If $\xi \in r\left(u^{\prime}\right)$, then by 5.5 there exists $\beta \in p\left(u^{\prime}\right)$ such that

$$
\xi=\beta-p_{1}(\beta)+q p_{1}(\beta) .
$$

Then $\xi-\beta=q p_{1}(\beta)-p_{1}(\beta) \in u$. Also, the element $\alpha=\beta-p_{1}(\beta)$ belongs to $a_{0}, \alpha-\beta=-p_{1}(\beta) \in a_{0,1}$, and $\xi-\alpha=q p_{1}(\beta) \in q\left(u^{\prime}\right)$. Thus (i) holds.

Conversely, if (i) holds, then

$$
\begin{gathered}
\beta=p(\xi), \quad \beta-\alpha=p_{1}(\beta), \quad \xi-\alpha=q(\beta-\alpha)=q p_{1}(\beta), \\
\xi=\beta-p_{1}(\beta)+q p_{1}(\beta)=r(\beta) \in r\left(u^{\prime}\right) .
\end{gathered}
$$


Theorem 5.7. If $p \in P_{0}$ and $f \in T_{0}$, then $p \odot f \in T_{0}$.

Proof. Let $q, r$ be as in 5.5, and let $x=p\left(u^{\prime}\right), y=q\left(u^{\prime}\right)$. Then $x, y$ are points and belong to $B^{\prime}$. Let

$$
y_{0}=\left[\left(a_{0}+x\right) a_{0,1}+u\right] y, \quad y_{1}=y_{0}\left(a_{0}+a_{1}\right) .
$$

By $4.3, y_{0} \in B^{\prime}$, and therefore $y_{1} \in B^{\prime}$. It is easy to check that

$$
y_{1}=\left[\left(a_{0}+x\right) a_{0,1}+a_{1}\right] y \text {. }
$$

Choose $y_{2} \in B^{\prime}$ with $y_{0}=y_{1}+y_{2}$, and let

$$
\begin{aligned}
y_{3} & =\left(a_{1}+a_{2}\right)\left(a_{0,2}+y_{1}\right), & x_{0} & =\left(a_{0}+a_{2}\right)\left(a_{1,2}+x\right), \\
y_{4} & =\left(a_{0,1}+y_{2}\right) u, & z_{0} & =\left(a_{0}+a_{1}\right)\left(x_{0}+y_{3}\right), \\
z_{1} & =\left(a_{0}+y_{2}\right)\left(x+y_{4}\right), & z & =z_{0}+z_{1} .
\end{aligned}
$$

Clearly $z \in B^{\prime}$. The proof will therefore be complete if it is shown that $(p \odot f)\left(a_{0}\right)=z$.

For any $\xi \in z_{0}$ there exists $\eta$ such that

$$
\eta \in x_{0}, \quad \xi-\eta \in y_{3} .
$$

Since $x_{0}=\left(a_{0}+a_{0,2}\right)\left(x+a_{1,2}\right)$, there exist $\alpha, \beta$ such that

$$
\alpha \in a_{0}, \quad \eta-\alpha \in a_{0,2}, \quad \beta \in x, \quad \eta-\beta \in a_{1,2} .
$$

It follows that

$\xi-\alpha \in\left(a_{0}+z_{0}\right)\left(y_{3}+a_{0,2}\right) \leqq\left(a_{0}+a_{1}\right)\left(a_{0,2}+y_{1}\right)=\left(a_{0}+a_{1}\right) a_{0,2}+y_{1}=y_{1}$,

$\xi-\beta \in\left(z_{0}+x\right)\left(y_{3}+a_{1,2}\right) \leqq\left(a_{0}+a_{1}\right)\left(a_{1}+a_{2}\right)=a_{1}$,

$\alpha-\beta \in\left(a_{0}+x\right)\left(a_{0,2}+a_{12}\right) \leqq\left(a_{0}+a_{1}\right)\left(a_{0,1}+a_{12}\right)=a_{0,1}$.

Thus the condition $\xi \in z_{0}$ implies that there exist $\alpha, \beta$ such that

(1) $\quad \alpha \in a_{0}, \quad \xi-\alpha \in y, \quad \beta \in x, \quad \xi-\beta \in u, \quad \alpha-\beta \in a_{0,1}$,

and therefore that $\xi \in(p \odot f)\left(a_{0}\right)$. Consequently $z_{0} \leqq(p \odot f)\left(a_{0}\right)$.

If $\xi \in z_{1}$, then there exist $\alpha, \beta$ such that

$$
\alpha \in a_{0}, \quad \xi-\alpha \in y_{2}, \quad \beta \in x, \quad \xi-\beta \in y_{4},
$$

and therefore

$\alpha-\beta \in\left(a_{0}+x\right)\left(y_{2}+y_{4}\right) \leqq\left(a_{0}+a_{1}\right)\left(a_{0,1}+y_{2}\right)=a_{0,1}+\left(a_{0}+a_{1}\right) y_{2}=a_{0,1}$.

Therefore (1) holds in this case also. Thus $z_{1} \leqq(p \odot f)\left(a_{0}\right), z \leqq(p \odot f)\left(a_{0}\right)$.

Conversely, if $\xi \in(p \odot f)\left(a_{0}\right)$, then (1) holds for some $\alpha, \beta$, and hence

$$
\alpha-\beta \in\left(a_{0}+x\right) a_{0,1}, \quad \xi-\alpha \in\left[\left(a_{0}+x\right) a_{0,1}+u\right] y=y_{0} .
$$

Thus there exist $\lambda_{1}, \lambda_{2}$ such that 


$$
\lambda_{1} \in y_{1}, \quad \lambda_{2} \in y_{2}, \quad \xi-\alpha=\lambda_{1}+\lambda_{2} .
$$

Therefore, for some $\mu_{1}$,

$$
\mu_{1} \in\left(a_{0}+x\right) a_{0,1}, \quad \lambda_{1}-\mu_{1} \in a_{1},
$$

and for some $\nu_{1}$,

$$
\nu_{1} \in a_{0} \text { and } \mu_{1}-\nu_{1} \in x .
$$

Let

$$
\alpha_{1}=-\nu_{1}, \quad \beta_{1}=\mu_{1}-\nu_{1}, \quad \xi_{1}=\lambda_{1}-\nu_{1}
$$

Then

(2) $\alpha_{1} \in a_{0}, \quad \xi_{1}-\alpha_{1} \in y_{1}, \quad \beta_{1} \in x, \quad \xi_{1}-\beta_{1} \in a_{1}, \quad \alpha_{1}-\beta_{1} \in a_{0,1}$.

It follows that, for some $\eta$,

$$
\eta \in a_{0,2}, \quad \beta_{1}-\alpha_{1}-\eta \in a_{1,2} .
$$

Consequently

$$
\begin{aligned}
\eta+\alpha_{1} \in\left(a_{0}+a_{0,2}\right)\left(a_{1,2}+x\right) & =\left(a_{0}+a_{2}\right)\left(a_{1,2}+x\right)=x_{0}, \\
\xi_{1}-\alpha_{1}-\eta \in\left(a_{1}+a_{1,2}\right)\left(a_{\theta, 2}+y_{1}\right) & =\left(a_{1}+a_{2}\right)\left(a_{0,2}+y_{1}\right)=y_{3}, \\
\xi_{1} \in\left(a_{0}+y_{1}\right)\left(x_{0}+y_{3}\right) & \leqq z_{0} .
\end{aligned}
$$

Let

$$
\xi_{2}=\xi-\xi_{1}, \quad \alpha_{2}=\alpha-\alpha_{1}, \quad \beta_{2}=\beta-\beta_{1} .
$$

Then by (1) and (2),

$\alpha_{2} \in a_{0}, \quad \xi_{2}-\alpha_{2}=\lambda_{2} \in y_{2}, \quad \beta_{2} \in x, \quad \xi_{2}-\beta_{2} \in u, \quad \alpha_{2}-\beta_{2} \in a_{0,1}$, and hence

$$
\xi_{2}-\beta_{2} \in\left(a_{0,1}+y_{2}\right) u=y_{4}, \quad \xi_{2} \in\left(a_{0}+y_{2}\right)\left(x+y_{4}\right)=z_{1} .
$$

Thus

$$
\xi=\xi_{1}+\xi_{2} \in z_{0}+z_{1}=z .
$$

The groups $(p \odot f)\left(a_{0}\right)$ and $z$ are therefore equal, and the proof is complete.

Lemмa 5.8. If $s, t$ are special endomorphisms, then so is $s+t$. In fact, if $p$ and $q$ are the special projections with which $s$ and $t$ are associated, then

$$
r=p-p_{0}+q
$$

is a special projection and $s+t$ is the endomorphism associated with $r$.

Proof. Clearly $p r=p$ and $r p=r$, so that $r \in P$. Furthermore $r\left(u^{\prime}\right) \leqq p\left(u^{\prime}\right)$ $+p_{0}\left(u^{\prime}\right)+q\left(u^{\prime}\right) \leqq a_{0}+a_{1}$. By $2.7, p f^{i}=p$. Therefore 


$$
\begin{aligned}
f^{8+t} & =f^{8} f^{t}=\left(1+(f-1) p_{1} p\right) f^{t}=f^{t}+(f-1) p_{1} p \\
& =1+(f-1) p_{1}(p+q)=1+(f-1) p_{1} r,
\end{aligned}
$$

because $p_{1} p_{0}=0$. By $5.7, f^{*}$ and $f^{t}$ are admissible translations, and therefore so is $f^{s} f^{t}$ by 4.5. In particular, if $h$ is the translation such that $h\left(a_{0}\right)=a_{0,1}$, then $h$ is admissible, therefore $h^{s+t}$ is admissible, so that $h^{s+t}\left(a_{0}\right) \in B^{\prime}$. But $h^{s+t}\left(a_{0}\right)$ $=r\left(u^{\prime}\right)$. Consequently $r$ is special.

Lemma 5.9. If $s$ is a special endomorphism, then so is $-s$. In fact, if $s$ is associated with the special projection $p$, then $r=2 p_{0}-p$ is a special projection and $-s$ is associated with $r$.

Proof. Clearly $r p=r$ and $p r=p$, so that $r \in P$. Also $r\left(u^{\prime}\right) \leqq p_{0}\left(u^{\prime}\right)+p\left(u^{\prime}\right)$ $\leqq a_{0}+a_{1}$. Therefore, for any translation $f$,

$$
f^{\prime}=1+(f-1) p_{1} r
$$

is a translation by 3.4. Furthermore,

$$
f^{\prime} f^{s}=1+(f-1) p_{1}(r+p)=1,
$$

because $r+p=2 p_{0}$ and $p_{1} p_{0}=0$. Thus $f^{\prime}=f^{-8}$.

To complete the proof it must be shown that $r$ is special. This will be done by proving that if $x=p\left(u^{\prime}\right)$, then $r\left(u^{\prime}\right)$ is equal to the element

$$
x^{\prime}=\left(\left(\left(x+a_{2}\right)\left(a_{0,2}+a_{1}\right)+a_{0}\right) u+a_{0,2}\right)\left(a_{0}+a_{1}\right) .
$$

In order to prove this it suffices to show that $r\left(u^{\prime}\right) \leqq x^{\prime}$ and $x^{\prime} u=0$.

If $\xi \in r\left(u^{\prime}\right)$, then $\xi=r(\alpha)$ for some $\alpha \in a_{0}$, because $r=r p_{0}$. There exists $\lambda$ such that

$$
\lambda \in a_{0,2} \text { and } \alpha-\lambda \in a_{2} .
$$

Also,

$$
p(\alpha) \in x, \quad \alpha-p(\alpha) \in\left(a_{0}+x\right) u \leqq\left(a_{0}+a_{1}\right) u=a_{1} .
$$

Consequently

$$
\begin{aligned}
& \alpha-\lambda-p(\alpha) \in\left(x+a_{2}\right)\left(a_{0,2}+a_{1}\right), \\
& 2 \alpha-\lambda-p(\alpha) \in\left(\left(x+a_{2}\right)\left(a_{0,2}+a_{1}\right)+a_{0}\right)\left(a_{1}+a_{2}\right), \\
& \xi=2 \alpha-p(\alpha) \in\left[\left(\left(x+a_{2}\right)\left(a_{0,2}+a_{1}\right)+a_{0}\right)\left(a_{1}+a_{2}\right)+a_{0,2}\right]\left(a_{0}+x\right) \leqq x^{\prime} .
\end{aligned}
$$

Let

$$
y=\left(\left(x+a_{2}\right)\left(a_{0,2}+a_{1}\right)+a_{0}\right)\left(a_{1}+a_{2}\right) .
$$

Then $x^{\prime} u=\left(y+a_{0,2}\right) a_{1}$. Now $\left(x, a_{1}, a_{2}\right) \perp$ because $x u=0$. Therefore $\left(x+a_{2}\right)\left(a_{0,2}+a_{1}\right) a_{1}=0$. Also, 


$$
\left(\left(x+a_{2}\right)\left(a_{0,2}+a_{1}\right)+a_{1}\right) a_{0} \leqq\left(a_{0,2}+a_{1}\right) a_{0}=0,
$$

so that $\left(\left(x+a_{2}\right)\left(a_{0,2}+a_{1}\right), a_{0}, a_{1}\right) \perp$. Therefore $y a_{1}=0$. Furthermore, $\left(y+a_{1}\right) a_{0,2}$ $\leqq\left(a_{1}+a_{2}\right) a_{0,2}=0$. Therefore $\left(y, a_{0,2}, a_{1}\right) \perp$, so that $x^{\prime} u=0$, as was to be shown.

LemMa 5.10. If $s$ and $t$ are special endomorphisms, then so is ts. In fact, if $p$ and $q$ are the special projections with which $s$ and $t$ are associated, then

$$
r=p-p_{1} p+q p_{1} p
$$

is a special projection and ts is the endomorphism associated with $r$.

Proof. From the fact that $p p=p$ and $p q=p$ it follows that $r p=r$ and $p r=p$, hence $r \in P$. Also, $r\left(u^{\prime}\right) \leqq a_{0}+a_{1}$ because the range of each of the functions $p, p_{1}, q$ is contained in $a_{0}+a_{1}$. Furthermore, for all $f \in T$,

$$
\begin{aligned}
\left(f^{t}\right)^{s} & =1+\left(f^{t}-1\right) p_{1} p=1+\left(1+(f-1) p_{1} q-1\right) p_{1} p \\
& =1+(f-1) p_{1} q p_{1} p=1+(f-1) p_{1} r,
\end{aligned}
$$

because $p_{1} r=p_{1} p-p_{1} p_{1} p+p_{1} q p_{1} p=p_{1} q p_{1} p$. By 5.7 , the translation

$$
f^{t s}=\left(f^{t}\right)^{s}=p \odot(q \odot f)
$$

is admissible. Therefore $r\left(u^{\prime}\right)=f^{t_{s}}\left(a_{0}\right)$ is a member of $B^{\prime}$, and $r$ is a special projection.

THEOREM 5.11. $E_{0}$ is a subring of $E$ containing the identity element of $E$.

Proof. The closure of $E_{0}$ under the ring operations was proved in 5.8, 5.9 and 5.10. Let $p$ be the member of $P_{0}$ such that $p\left(u^{\prime}\right)=a_{0,1}$. Then $p_{1} p=p$, and it follows that for any translation $f$,

$$
p \odot f=1+(f-1) p=1+f p-p,
$$

hence $p \odot f=f$ by 2.4. Thus the endomorphism 1 is associated with $p$ and is therefore special.

6. The regularity of $E_{0}$. The next theorem is an improved version of the existence Theorem 3.4 for the special case in which the translations involved are admissible. The regularity of $E_{0}$ is an easy consequence of this result.

THEOREM 6.1. If $f, f^{\prime}$ are admissible translations and $\pi\left(f^{\prime}\right) \leqq \pi(f)$, then there exists a special endomorphism such that $f^{\prime}=f^{s}$.

Proof. Let

$$
y=f\left(a_{0}\right), \quad z=f^{\prime}\left(a_{0}\right), \quad y_{0}=a_{0} y, \quad z_{0}=a_{0} z, \quad \bar{y}=\left(a_{0}+z\right) y,
$$

choose $y_{1}, y_{2}, \bar{z} \in B^{\prime}$ such that

$$
\left(a_{0}+a_{1}\right) \bar{y}=y_{0}+y_{1}, \quad \bar{y}=y_{0}+y_{1}+y_{2}, \quad z=z_{0}+\bar{z},
$$

and let 


$$
\begin{array}{ll}
z_{1}=\left(a_{0}+y_{1}\right) \bar{z}, & z_{2}=\left(a_{0}+y_{2}\right) \bar{z}, \\
v_{1}=\left(y_{1}+a_{0,2}\right) u, & w=\left(z_{1}+v_{1}\right)\left(a_{0}+a_{2}\right), \\
x_{1}=\left(w+a_{1,2}\right)\left(a_{0}+a_{1}\right), & v_{2}=\left(y_{2}+a_{0,1}\right) u, \\
x_{2}=\left(z_{2}+v_{2}\right)\left(a_{0}+a_{1}\right), & x=z_{0}+x_{1}+x_{2} .
\end{array}
$$

It will be shown that $x$ is a point, and that $p \odot f=f^{\prime}$ where $p$ is the member of $P_{0}$ that maps $u^{\prime}$ onto $x$.

First observe that

$$
a_{0}+y_{1}+y_{2}=a_{0}+\bar{y}=a_{0}+z=a_{0}+\bar{z},
$$

and therefore

$$
\begin{aligned}
a_{0} & +z_{1}=a_{0}+y_{1}, \quad a_{0}+z_{2}=a_{0}+y_{2}, \\
z_{1}+z_{2} & =\left(a_{0}+y_{1}\right) \bar{z}+\left(a_{0}+y_{2}\right) \bar{z}=\left[\left(a_{0}+y_{1}\right) \bar{z}+a_{0}+y_{2}\right] \bar{z} \\
& =\left[\left(a_{0}+y_{1}\right)\left(a_{0}+\bar{z}\right)+y_{2}\right] \bar{z}=\left(a_{0}+y_{1}+y_{2}\right) \bar{z}=\bar{z} \\
z_{0}+z_{1}+z_{2} & =z .
\end{aligned}
$$

Next it will be shown that

$$
x_{1}+a_{1}=z_{1}+a_{1} .
$$

In fact,

$$
\begin{aligned}
x_{1}+a_{1} & =\left(a_{0}+a_{1}\right)\left(w+a_{1,2}+a_{1}\right)=\left(a_{0}+a_{1}\right)\left(w+a_{1}+a_{2}\right), \\
w+a_{2} & =\left(z_{1}+v_{1}+a_{2}\right)\left(a_{0}+a_{2}\right), \\
v_{1}+a_{2} & =\left(y_{1}+a_{0,2}+a_{2}\right) u=\left(y_{1}+a_{0}+a_{2}\right) u \\
& =\left(y_{1}+a_{0}\right) u+a_{2}=\left(y_{1}+a_{0}\right) a_{1}+a_{2}, \\
w+a_{2} & =\left[z_{1}+\left(y_{1}+a_{0}\right) a_{1}+a_{2}\right]\left(a_{0}+a_{2}\right) \\
& =\left[z_{1}+\left(y_{1}+a_{0}\right) a_{1}\right]\left(a_{0}+a_{2}\right)+a_{2} \\
& =\left(z_{1}+a_{1}\right)\left(y_{1}+a_{0}\right)\left(a_{0}+a_{2}\right)+a_{2} \\
& =\left(z_{1}+a_{1}\right)\left[a_{0}+y_{1}\left(a_{0}+a_{2}\right)\right]+a_{2} \\
& =\left(z_{1}+a_{1}\right) a_{0}+a_{2},
\end{aligned}
$$

and therefore

$$
\begin{aligned}
x_{1}+a_{1} & =\left(a_{0}+a_{1}\right)\left[\left(z_{1}+a_{1}\right) a_{0}+a_{1}+a_{2}\right] \\
& =\left(z_{1}+a_{1}\right) a_{0}+a_{1}+\left(a_{0}+a_{1}\right) a_{2} \\
& =\left(z_{1}+a_{1}\right)\left(a_{0}+a_{1}\right)=z_{1}+a_{1},
\end{aligned}
$$

because $z_{1} \leqq a_{0}+a_{1}$.

It will now be shown that 


$$
x_{2}+u=z_{2}+u \text {. }
$$

In fact,

$$
\begin{aligned}
x_{2}+a_{1} & =\left(a_{0}+a_{1}\right)\left(z_{2}+v_{2}+a_{1}\right), \\
v_{2}+a_{1} & =\left(y_{2}+a_{0,1}+a_{1}\right) u=\left(y_{2}+a_{0}+a_{1}\right) u, \\
z_{2}+v_{2}+a_{1} & =\left(y_{2}+a_{0}+a_{1}\right)\left(z_{2}+u\right) \\
x_{2}+a_{1} & =\left(a_{0}+a_{1}\right)\left(z_{2}+u\right) \\
x_{2}+u & =\left(a_{0}+a_{1}\right)\left(z_{2}+u\right)+u \\
& =\left(a_{0}+a_{1}+u\right)\left(z_{2}+u\right)=z_{2}+u .
\end{aligned}
$$

Consequently,

$$
x+u=z_{0}+x_{1}+x_{2}+u=z_{0}+z_{1}+z_{2}+u=z+u=u^{\prime} .
$$

Clearly $\left(a_{0}, y_{1}, y_{2}\right) \perp$. Therefore

$$
\left(a_{0}+z_{1}\right)\left(a_{0}+z_{2}\right)=\left(a_{0}+y_{1}\right)\left(a_{0}+y_{2}\right)=a_{0} .
$$

Inasmuch as $a_{0} z=0$, hence $a_{0} z_{1}=a_{0} z_{2}=0$, it follows that $\left(a_{0}, z_{1}, z_{2}\right) \perp$. Consequently $z_{1} z_{2}=0$. This, together with the fact that $\left(z_{1}+z_{2}\right) u \leqq z u=0$, implies that $\left(u, z_{1}, z_{2}\right) \perp$. It will now be shown that $\left(u, x_{1}, x_{2}\right) \perp$. First observe that

$$
\left(u+x_{1}\right)\left(u+x_{2}\right)=\left(u+z_{1}\right)\left(u+z_{2}\right)=u,
$$

so that the three elements $u, x_{1}, x_{2}$ generate a distributive lattice. It is therefore sufficient to show that they are pairwise disjoint. Now

$$
\begin{aligned}
x_{1} u & =\left(w+a_{1,2}\right) a_{1}, \\
\left(a_{1}+a_{1,2}\right) w & =\left(a_{1}+a_{2}\right)\left(a_{0}+a_{2}\right)\left(z_{1}+v_{1}\right)=a_{2}\left(z_{1}+v_{1}\right), \\
a_{2} v_{1} & =a_{2}\left(y_{1}+a_{0,2}\right), \\
\left(a_{2}+a_{0,2}\right) y_{1} & =\left(a_{0}+a_{2}\right) y_{1}=0 .
\end{aligned}
$$

Therefore $\left(a_{2}, a_{0,2}, y_{1}\right) \perp, a_{2} v_{1}=0$. Since

$$
\left(a_{2}+v_{1}\right) z_{1} \leqq u z_{1}=0,
$$

it follows that $\left(a_{2}, z_{1}, v_{1}\right) \perp$, and therefore $\left(a_{1}+a_{1,2}\right) w=0,\left(a_{1}, a_{1,2}, w\right) \perp, x_{1} u=0$. Furthermore

$$
\begin{aligned}
x_{2} u & =\left(z_{2}+v_{2}\right) a_{1}, \\
a_{1} v_{2} & =\left(y_{2}+a_{0,1}\right) a_{1}, \\
\left(a_{1}+a_{0,1}\right) y_{2} & =\left(a_{0}+a_{1}\right) y_{2}=0,
\end{aligned}
$$

so that $\left(a_{1}, a_{0,1}, y_{2}\right) \perp, a_{1} v_{2}=0$. Since also,

$$
\left(a_{1}+v_{2}\right) z_{2} \leqq u z_{2}=0,
$$


this implies that $\left(a_{1}, z_{2}, v_{2}\right) \perp$ and therefore $x_{2} u=0$. This completes the proof of the assertion that $\left(u, x_{1}, x_{2}\right) \perp$. Moreover

$$
z_{0}\left(u+x_{1}+x_{2}\right)=a_{0} z\left(u+z_{1}+z_{2}\right)=a_{0}\left(z_{1}+z_{2}\right)=0,
$$

so that $\left(u, z_{0}, x_{1}, x_{2}\right) \perp$. Since it has already been proved that $x+u=u^{\prime}$, this shows that the element $x=z_{0}+x_{1}+x_{2}$ is a point.

The proof will now be completed by showing that $(p \odot f)\left(a_{0}\right)=z$. Since both $z$ and $(p \odot f)\left(a_{0}\right)$ are points, it suffices to prove that $z \leqq(p \odot f)\left(a_{0}\right)$. According to 5.6 this means that for any $\xi \in z$ there exist $\alpha, \beta$ such that

$$
\alpha \in a_{0}, \quad \xi-\alpha \in y, \quad \beta \in x, \quad \xi-\beta \in u, \quad \alpha-\beta \in a_{0,1} .
$$

It is clearly sufficient to prove this under the assumption that $\xi$ belongs to one of the groups $z_{i}, i=0,1,2$. If $\xi \in z_{0}$, then (1) holds with $\alpha=\beta=\xi$. It may therefore be assumed that $\xi \in z_{1}$ or $\xi \in z_{2}$.

Suppose $\xi \in z_{1}$. Since $z_{1} \leqq a_{0}+y_{1}$, there exists $\alpha$ such that

$$
\alpha \in a_{0} \text { and } \xi-\alpha \in y_{1} \text {. }
$$

Observe that $z_{1} \leqq v_{1}+w$. In fact,

$$
\begin{aligned}
v_{1}+w & =\left(z_{1}+v_{1}\right)\left(a_{0}+a_{2}+v_{1}\right), \\
a_{2}+v_{1} & =\left(y_{1}+a_{2}+a_{0,2}\right) u=\left(y_{1}+a_{0}+a_{2}\right) u=\left(z_{1}+a_{0}+a_{2}\right) u, \\
a_{0}+a_{2}+v_{1} & =z_{1}+a_{0}+a_{2},
\end{aligned}
$$

whence the desired inclusion follows. Consequently there exists $\lambda$ such that

$$
\lambda \in w \text { and } \xi-\lambda \in v_{1} \text {. }
$$

Since

$$
\begin{aligned}
x_{1}+a_{1,2} & =\left(w+a_{1,2}\right)\left(a_{0}+a_{1}+a_{1,2}\right)=\left(w+a_{1,2}\right)\left(a_{0}+a_{1}+a_{2}\right) \\
& =w+a_{1,2} \geqq w,
\end{aligned}
$$

there exists $\beta$ such that

$$
\beta \in x_{1} \text { and } \lambda-\beta \in a_{1,2} .
$$

It follows that

$$
\begin{aligned}
\xi-\beta \in\left(v_{1}+a_{1,2}\right)\left(z_{1}+x_{1}\right) & \leqq\left(v_{1}+a_{1,2}\right)\left(a_{0}+a_{1}\right) \leqq u\left(a_{0}+a_{1}\right)=a_{1}, \\
\lambda-\alpha \in\left(y_{1}+v_{1}\right)\left(w+a_{0}\right) & \leqq\left(y_{1}+a_{0,2}\right)\left(a_{0}+a_{2}\right)=a_{0,2}+y_{1}\left(a_{0}+a_{2}\right)=a_{0,2}, \\
\alpha-\beta \in\left(a_{0,2}+a_{1,2}\right)\left(a_{0}+x_{1}\right) & \leqq\left(a_{0,2}+a_{1,2}\right)\left(a_{0}+a_{1}\right)=a_{0,1},
\end{aligned}
$$

so that (1) holds in this case.

Now suppose $\xi \in z_{2}$. Since $z_{2} \leqq a_{0}+y_{2}$ there exists $\alpha$ such that

$$
\alpha \in a_{0} \text { and } \xi-\alpha \in y_{2} \text {. }
$$

Observe that $x_{2}+v_{2} \geqq z_{2}$. In fact, 


$$
\begin{aligned}
x_{2}+v_{2} & =\left(z_{2}+v_{2}\right)\left(v_{2}+a_{0}+a_{1}\right), \\
v_{2}+a_{1} & =\left(y_{2}+a_{0,1}+a_{1}\right) u=\left(y_{2}+a_{0}+a_{1}\right) u=\left(z_{2}+a_{0}+a_{1}\right) u, \\
v_{2}+a_{0}+a_{1} & =z_{2}+a_{0}+a_{1},
\end{aligned}
$$

whence the desired inequality follows. Consequently there exists $\beta$ such that

$$
\beta \in x_{2} \text { and } \xi-\beta \in v_{2} \leqq u \text {. }
$$

It follows that

$\alpha-\beta \in\left(a_{0}+x_{2}\right)\left(y_{2}+v_{2}\right) \leqq\left(a_{0}+a_{1}\right)\left(y_{2}+a_{0,1}\right)=\left(a_{0}+a_{1}\right) y_{2}=a_{0,1}=a_{0,1}$.

Thus (1) is seen to hold in this case also.

This completes the proof.

Corollary 6.2. For any $f, g \in T_{0}, f^{E_{0}} \subseteq g^{E_{0}}$ if and only if $\pi(f) \leqq \pi(g)$.

LEMMA 6.3. Suppose the special endomorphisms $s, t, t^{\prime}$ are associated with the special projections $p, q, q^{\prime}$, and let $f=1-p_{0}+q, f^{\prime}=1-p_{0}+q^{\prime}$. Then

$$
t s=t^{\prime} \text { if and only if } p \odot f=f^{\prime} .
$$

Proof. By 5.10, $t s=t^{\prime}$ if and only if $q^{\prime}=p-p_{1} p+q p_{1} p$, and by substituting into 5.4 it is found that $p \odot f=f^{\prime}$ if and only if $q^{\prime}=p_{0}+q p_{1} p-p_{0} p_{1} p$. The lemma is therefore a consequence of the observation, made at the end of the discussion that precedes Theorem 5.4, that $p_{0} p_{1} p=p_{0}-p+p_{1} p$.

COROLlary 6.4. Suppose the special endomorphisms $t, t^{\prime}$ are associated with the special projections $q, q^{\prime}$. Then $t E_{0}=t^{\prime} E_{0}$ if and only if $a_{0}+q\left(u^{\prime}\right)=a_{0}+q^{\prime}\left(u^{\prime}\right)$.

Proof. If $f, f^{\prime}$ are defined as in 6.3, then it follows from 3.2 that the conditions $a_{0}+q\left(u^{\prime}\right)=a_{0}+q^{\prime}\left(u^{\prime}\right)$ and $\pi(f)=\pi\left(f^{\prime}\right)$ are equivalent. Together with 6.1 and 6.3 this yields the conclusion.

LEMMA 6.5. Suppose the special endomorphism $s$ is associated with the special projection s. If there exist $y, z \in B^{\prime}$ such that $p\left(u^{\prime}\right)=y+z, y \leqq a_{0}$ and $z \leqq a_{0,1}$, then $s$ is idempotent.

Proof. For any translation $f$, $\left(f^{*}\right)^{*}=1+\left(f^{*}-1\right) p_{1} p=1+\left(1+(f-1) p_{1} p-1\right) p_{1} p=1+(f-1) p_{1} p p_{1} p$. If $\alpha \in y$, then $p_{1}(\alpha)=0$, hence $p_{1} p p_{1}(\alpha)=p_{1}(\alpha)$. If $\alpha \in z$, then $p_{1}(\alpha)=\alpha$ and $p(\alpha)=\alpha$, hence $p_{1} p p_{1}(\alpha)=p_{1}(\alpha)$. Consequently $p_{1} p p_{1}$ and $p_{1}$ agree on $p\left(u^{\prime}\right)$, so that $p_{1} p_{1} p=p_{1} p,\left(f^{*}\right)^{s}=f^{*}$.

THEOREM 6.6. $E_{0}$ is a regular ring.

Proof. It is known (von Neumann [9, Part II, Theorem 2.2]) that a ring with identity is regular if and only if every principal right ideal is generated 
by an idempotent element. Consider therefore a principal right ideal $t E_{0}$, and let $q$ be the special projection with which $t$ is associated. Also let $x=q\left(u^{\prime}\right)$, $z=\left(a_{0}+x\right) a_{0,1}$, and choose $y \in B^{\prime}$ such that $y \leqq a_{0}$ and $a_{0}=y+\left(a_{1}+z\right) a_{0}$. Then

$$
a_{0}+a_{1}=y+\left(a_{1}+z\right) a_{0}+a_{1}=y+z+a_{1} \text {. }
$$

Since also, $\left(a_{1}, z, y\right) \perp$, it follows that $y+z$ is a point. Let $q^{\prime}$ be the member of $P_{0}$ such that $q^{\prime}\left(u^{\prime}\right)=y+z$, and let $t^{\prime}$ be the special endomorphism associated with $q^{\prime}$. Then $t^{\prime}$ is idempotent by 6.5 . Furthermore,

$$
a_{0}+q^{\prime}\left(u^{\prime}\right)=a_{0}+y+z=a_{0}+z=a_{0}+x=a_{0}+q\left(u^{\prime}\right) .
$$

Consequently $t E_{0}=t^{\prime} E_{0}$ by 6.4 .

7. Finitely generated submodules of $T_{0}$. The group $T_{0}$ of all admissible translations may be regarded as a module over the ring $E_{0}$ of special endomorphisms. With each subgroup $b$ of $u$ there is associated a submodule of $T_{0}$, consisting of all those special translations whose trace is contained in $b$. In general this is not a lattice isomorphism (or even a homomorphism) between the subgroups of $u$ and the submodules of $T_{0}$. However, as will be shown in this section, $T_{0}$ is locally projective, and the lattice of all finitely generated submodules of $T_{0}$ is isomorphic to a certain ideal $C$ of $B^{\prime} u$. The representation theorem, formulated in the next section, is simply the specialization of this result to the particular case in which $C=B^{\prime} u$.

The following notation will be fixed throughout this section:

Definition 7.1. For any subgroup $b$ of $u, F(b)$ is the set of all admissible translations $f$ with $\pi(f) \leqq b$.

Definition 7.2. $B y C$ will be meant the ideal of $B^{\prime} u$ that is generated by the set of all elements $b \in B^{\prime} u$ such that $b$ is perspective in $B^{\prime}$ to some element $x \in B^{\prime}$ with $x \leqq a_{0}$.

Corollary 7.3. For any subgroup $b$ of $u, F(b)$ is a submodule of $T_{0}$.

Proof. From the fact that $\pi(1)=0$ it follows that $1 \in F(b)$. By 2.14 and $2.15, F(b)$ is a subgroup of $T_{0}$. Finally, if $f \in F(b)$ and $s \in E_{0}$, then $\pi\left(f^{s}\right)$ $\leqq \pi(f) \leqq b$, so that $f^{\circ} \in F(b)$.

Corollary 7.4. For any subgroups $b, c$ of $u$,

$$
F(b c)=F(b) \cap F(c) .
$$

LеммA 7.5. For any subgroups $b, c$ of $u$, if $b, c \in B^{\prime}$, then $F(b+c)=F(b) F(c)\left({ }^{2}\right)$.

Proof. It is clearly sufficient to consider the case in which $b c=0$, and to show that in this case $F(b+c) \leqq F(b) F(c)$. Assuming that $f \in F(b+c)$, let

Then

$$
x=f\left(a_{0}\right), \quad y=\left(a_{0}+b\right)(x+c), \quad z=\left(a_{0}+c\right)(x+b) .
$$

(2) Since the group operation in $T_{0}$ is written as multiplication, the lattice sum of the modules $F(b)$ and $F(c)$ is the complex product, $F(b) F(c)$. 


$$
\begin{aligned}
y+u & =\left(a_{0}+b+c\right)(x+c)+u=x+c+u=u^{\prime}, \\
y u & =\left(a_{0}+b\right) u(x+c) u=\left(a_{0} u+b\right)(x u+c)=b c=0,
\end{aligned}
$$

so that $y$ is a point. Similarly $z$ is a point. Let $g$ and $h$ be the translations such that $g\left(a_{0}\right)=y$ and $h\left(a_{0}\right)=z$. Then $g, h$ are admissible by 4.4. Also,

$$
\pi(g)=\left(a_{0}+y\right) u \leqq\left(a_{0}+b\right) u=b,
$$

so that $g \in F(b)$. Similarly $h \in F(c)$, and the proof is therefore complete if it is shown that $f=g h$. In view of 2.8 it suffices to show that $x \geqq g h\left(a_{0}\right)=g(z)$.

Given $\xi \in z$, there exists $\beta$ such that

$$
\beta \in x \text { and } \xi-\beta \in b,
$$

and since

$$
y+c=\left(a_{0}+b+c\right)(x+c) \geqq x,
$$

there exists $\gamma$ such that

$$
\gamma \in y \text { and } \beta-\gamma \in c .
$$

Consequently

$$
\xi-\beta+\gamma \in(y+b)(z+c) \leqq\left(a_{0}+b\right)\left(a_{0}+c\right)=a_{0} .
$$

Since $\xi-\beta, \beta-\gamma \in u$, it follows that

$$
g(\xi)=g(\xi-\beta+\gamma)+g(\beta-\gamma)=\gamma+(\beta-\gamma)=\beta \in x .
$$

Thus $g(z) \leqq x$, as was to be shown.

Lемма 7.6. For every admissible translation $f, F(\pi(f))=f^{E_{0}}$.

Proof. Clearly $f \in F(\pi(f))$, and therefore $f^{E_{0}} \subseteq F(\pi(f))$. Conversely, if $g \in F(\pi(f))$, then $\pi(g) \leqq \pi(f)$, whence it follows by 6.1 that $g=f^{8}$ for some $s \in E_{0}$.

Lemma 7.7. For any $b \in B^{\prime} u$, if $b$ is perspective in $B^{\prime}$ to $a$ part of $a_{0}$, then $b=\pi(f)$ for some admissible translation $f$.

Proof. By hypothesis there exists $x \in B^{\prime}$ such that $x \leqq a_{0}$ and $x \sim b$ in $B^{\prime}$. Then for some $y \in B^{\prime}, x \dot{+} b=x \dot{+} y=y+b$. Consequently

$$
\begin{aligned}
y u & =y(x+b) u=y b=0, \\
y+u & =y+b+u=x+b+u=x+u, \\
(y+u) a_{0} & =(x+u) a_{0}=x .
\end{aligned}
$$

Choose $z \in B^{\prime}$ such that $a_{0}=x+z$. Then $y+z$ is a point. By 2.8 there exists a translation $f$ such that $f\left(a_{0}\right)=y+z$, and by $4.4 f$ is admissible. Furthermore

$$
\pi(f)=\left(a_{0}+y+z\right) u=\left(a_{0}+y\right) u=\left(a_{0}+b\right) u=b .
$$


Lemma 7.8. For every $b \in C, F(b)$ is a finitely generated submodule of $T_{0}$.

Proof. According to CM1.5, every element of $C$ is the sum of finitely many elements, each of which is perspective in $B^{\prime}$ to a part of $a_{0}$. By 7.6 and 7.7, $F$ maps each of these summands onto a cyclic submodule of $T_{0}$, whence the conclusion follows by 7.5 .

LEMMA 7.9. If $M$ is a finitely generated submodule of $T_{0}$, then $M=F(b)$ for some $b \in C$.

Proof. By 7.5 it suffices to consider the case in which $M$ is cyclic, say $M=f^{E_{0}}$ with $f \in T_{0}$. According to 7.6 the conclusion will follow if it is shown that the element $b=\pi(f)$ is perspective in $B^{\prime}$ to a part of $a_{0}$.

Since $a_{0}+b=a_{0}+f\left(a_{0}\right)$, there exists $y \in B^{\prime}$ such that $y \leqq f\left(a_{0}\right)$ and $a_{0}+b$ $=a_{0}+y$. Let $x=a_{0}(y+b)$. Then

$$
\begin{gathered}
x y=a_{0} y=0, \quad y b \leqq f\left(a_{0}\right) u=0, \\
x+y=\left(a_{0}+y\right)(y+b)=\left(a_{0}+b\right)(y+b)=y+b .
\end{gathered}
$$

Thus $x \sim b$ in $B^{\prime}$, as was to be shown.

The following notation has already been used, in the introduction to this paper.

Definition 7.10. If $M$ is a module over a ring $R$, then by $L(M, R)$ will be meant the set of all finitely generated submodules of $M$.

THEOREM 7.11. $L\left(T_{0}, E_{0}\right)$ is a relatively complemented modular lattice, and $F$ maps $C$ isomorphically onto $L\left(T_{0}, E_{0}\right)$.

Proof. By 7.3, 7.4 and 7.5, $F$ maps $B^{\prime} u$ homomorphically into the lattice of all submodules of $T_{0}$, and by 7.8 and $7.9, F$ maps $C$ onto $L\left(T_{0}, E_{0}\right)$. Therefore $L\left(T_{0}, E_{0}\right)$ is a relatively complemented modular lattice.

For any nonzero member $b$ of $C$ there exists $y \in B^{\prime}$ such that $0 \neq y \leqq b$ and $y$ is perspective in $B^{\prime}$ to a part of $a_{0}$. Hence, by $7.7, y=\pi(f)$ for some admissible translation $f$, and it follows that $1 \neq f \in F(b)$. The restriction of $F$ to $C$ is therefore an isomorphism.

The final step in the study of the module $T_{0}$ is based on some simple observations concerning locally projective modules over regular rings.

Definition 7.12. A module $M$ over a ring $R$ is said to be locally projective if and only if every finitely generated submodule of $M$ is projective.

COROLlARY 7.13. For a regular ring $R$, the following statements hold:

(i) Every projective module over $R$ is locally projective.

(ii) $A$ locally projective module over $R$ is projective if and only if it is a direct sum of cyclic modules.

Proof. By Kaplansky [8, Lemma 4] every finitely generated submodule of 
a projective module $M$ over $R$ is a direct summand of $M$, and is therefore projective. Hence (i) holds.

By Kaplansky [8, Theorem 4] every projective module over $R$ is a direct sum of cyclic modules. Hence the forward implication in (ii) holds. The backward implication follows from 7.12 and the fact that a direct sum of projective modules is projective.

COROLlaRy 7.14. If $M$ is a locally projective module over a regular ring $R$, then $L(M, R)$ is a relatively complemented modular lattice.

Proof. It clearly suffices to consider the case in which $M$ is finitely generated, and therefore projective. Since in this case $M$ is a direct summand of a finitely generated free module, it may be assumed that $M$ is free, and in this case the assertion is true by von Neumann [9, Part II, Chap. 2, Appendix 3].

It is not known whether, conversely, the assumption that $L(M, R)$ is a relatively complemented modular lattice implies that $M$ is locally projective; it seems unlikely that this is the case. In the proof of the local projectivity of $T_{0}$ use will be made of the fact that it contains a submodule isomorphic to $E_{0}$.

LEMмA 7.15. Every finitely generated submodule of a cyclic submodule of $T_{0}$ is cyclic.

Proof. Since a direct summand of a cyclic module is always cyclic, the lemma is an immediate consequence of 7.11 .

Lemma 7.16. For every admissible translation $f$, the set of all special endomorphisms $s$ with $f^{*}=1$ is a principal right ideal of $E_{0}$.

Proof. Let $h$ be the translation such that $h\left(a_{0}\right)=a_{0,1}$. If $s$ is a special endomorphism and $p$ is the special projection with which $s$ is associated, then $h^{s}\left(a_{0}\right)=p\left(u^{\prime}\right)$. Consequently $h^{s}=1$ if and only if $p\left(u^{\prime}\right)=a_{0}$ or, equivalently, $s=0$.

Next consider an admissible translation $f$ such that $f^{E_{0}} \cap h^{E_{0}}=1$. By 7.15 the module $(h f)^{E_{0}} \cap h^{E_{0}}$ is cyclic, say

$$
(h f)^{E_{0}} \cap h^{E_{0}}=g^{E_{0}}
$$

Therefore $g=(h f)^{t}=h^{t^{\prime}}$ for some $t, t^{\prime} \in E_{0}$. Consequently $f^{t}=h^{t^{\prime}-t} \in f^{E_{0}} \bigcap h^{E_{0}}$, whence it follows that $f^{t}=1$ and $t=t^{\prime}$. Furthermore, if $s \in E_{0}$ and $f^{s}=1$, then

$$
(h f)^{s}=h^{s} \in(h f)^{E_{0}} \cap h^{E_{0}}=g^{E_{0}} \text {. }
$$

Consequently $h^{s}=g^{z^{\prime}}=h^{t s^{\prime}}$ for some $s^{\prime} \in E_{0}$, and it follows that $s=t s^{\prime}$. Thus it has been shown that, in this case, $f^{s}=1$ if and only if $s \in t E_{0}$.

In the general case there exists a finitely generated submodule $M$ of $f^{E_{0}}$ such that

$$
f^{E_{0}} h^{E_{0}}=M h^{E_{0}} \quad \text { and } \quad M \cap h^{E_{0}}=1 .
$$


Consequently $f=f^{t} h^{\prime \prime}$ where $t, t^{\prime} \in E_{0}$ and $f^{t} \in M$, and in fact $M$ is generated by $f^{t}$. It follows that, for any $s \in E_{0}, f^{s}=1$ if and only if $f^{t s}=1$ and $t^{\prime} s=0$. By the special case considered above, the set of all solutions $s$ of the equation $f^{t s}=1$ is a principal right ideal of $E_{0}$, and since $E_{0}$ is a regular ring the same is true of the solutions of the equation $t^{\prime} s=0$. Thus the set of all solutions of the equation $f^{s}=1$ is the intersection of two principal right ideals of $E_{0}$, and is therefore a principal right ideal by the regularity of $E_{0}$.

TheOREM 7.17. $T_{0}$ is a locally projective module over $E_{0}$.

Proof. Every finitely generated submodule of $T_{0}$ is a direct sum of cyclic submodules, and it is therefore sufficient to show that every cyclic submodule of $T_{0}$ is projective. If the given module $M$ is spanned by the admissible translation $f$, then by 7.16 there exists an idempotent element $t \in E_{0}$ such that, for all $s \in E_{0}, f^{s}=1$ if and only if $s \in t E_{0}$. Consequently the correspondence $s \rightarrow f^{s}$ is an isomorphism of the $E_{0}$ module $(1-t) E_{0}$ onto the $E_{0}$ module $M$. Since $(1-t) E_{0}$ is a direct summand of the free $E_{0}$ module $E_{0}$, and is therefore projective, it follows that $M$ is projective.

8. The representation theorem. The concept of a large element was mentioned in the introduction:

Definition 8.1. An element d of a modular lattice $B$ is said to be large if and only if every member of $B$ is the sum of finitely many elements each of which is perspective to a part of $d$.

THEOREM 8.2. If in a relatively complemented Arguesian lattice $B$ there exists a large element $d$ such that $B d$ possesses a homogeneous 3-frame, then $B \cong L(M, R)$ for some locally projective module $M$ over a regular ring $R$.

Proof. By 1.1 it may be assumed that $B$ is a sublattice of the lattice of all subgroups of an Abelian group $v$. If $b$ is the given homogeneous 3-frame of $B d$, then by 1.2 there exist an Abelian group $u^{\prime}$ containing $v$ as a subgroup, a relatively complemented sublattice $B^{\prime}$ of the lattice $A^{\prime}$ of all subgroups of $u^{\prime}$, and a partial 3-frame $a$ of $A^{\prime}$ such that $a^{*}=v, a_{i}, a_{i, j} \in B^{\prime}$ for $i, j=0,1,2$, $a_{i} \sim b_{0}$ in $B^{\prime}$ for $i=0,1,2$, and $B^{\prime}=B^{\prime} a_{0}+B^{\prime} a_{1} \dot{+} B^{\prime} a_{2} \dot{+} B$. Let $u=a_{0} \dot{+} a_{1} \dot{+} a_{2} \dot{+} v$, and let $A$ be the lattice of all subgroups of $u$. The conditions 4.1(i)-(iv) are then satisfied and, in addition, $B=B^{\prime} a^{*}$. All the results of $\S \S 4-7$ therefore apply.

Next observe that $C=B^{\prime} u$, where $C$ is defined as in 7.2. In fact, by CM1.5, $C$ is the intersect of $B^{\prime} u$ and a neutral ideal $C^{\prime}$ of $B^{\prime}$. Since the elements $a_{i}$ are perspective to $b_{0}$, they belong to $C^{\prime}$. Also, since $d$ is a large element of $B$, every element of $B$ belongs to $C^{\prime}$. Therefore $C^{\prime}=B^{\prime}$ and $C=B^{\prime} u$.

By 7.11 the function $F$ defined in 7.1 maps $B^{\prime} u$ isomorphically onto the lattice of all finitely generated submodules of the locally projective module $T_{0}$ over the regular ring $E_{0}$. The submodule $M=F(v)$ of $T_{0}$ is also locally projective, and for any $x \in B^{\prime} u, F(x) \subseteq M$ if and only if $x \leqq v$. In fact, $x v \in B$ 
by 4.3, and hence $x=x v+y$ for some $y \in B^{\prime} u$. If now $F(x) \subseteq M$, then $F(y) \subseteq M$. Hence $f \in F(y)$ implies that $\pi(f) \leqq y v=0, f=1$. Thus $y=0, x \leqq v$. This shows that $F(x) \subseteq M$ implies that $x \leqq v$, and the opposite inclusion is obvious. Thus $F$ maps $B$ isomorphical'y onto the lattice of all finitely generated submodules of $M$.

COROLlARY 8.3. If in a relatively complemented modular lattice $B$ there exists a large element $d$ such that the ideal $B d$ possesses a homogeneous n-frame with $n \geqq 4$, then $B \cong L(M, R)$ for some locally projective module $M$ over a regular ring $R$.

Proof. It follows from the hypothesis that if $x$ is any element of $B$ with $d \leqq x$, then the ideal $B x$ of $B$ possesses a large partial $n$-frame $b$ with $b_{0}+b_{1}+\cdots+b_{n-1}=d$. Consequently, by CM2.1, each such ideal $B x$ is Arguesian. The lattice $B$ is thus the union of a directed family of Arguesian sublattices, and is therefore Arguesian. Reference to 8.2 completes the proof.

COROLlARY 8.4. If $B$ is a simple, relatively complemented modular lattice of dimension four or more, then $B \cong L(M, R)$ for some locally projective module $M$ over a regular ring $R$.

Proof. By CM2.2, every ideal of $B$ is simple. Consequently, if $x$ is any element of $B$ of dimension four or more, then by CM1.7, $B x$ possesses a large partial 4-frame $b$. Let $d=b_{0}+b_{1}+b_{2}+b_{3}$. Then the ideal $B d$ possesses a homogeneous 4-frame. Furthermore, the set $I$ of all those elements that are sums of finitely many elements, each of which is perspective to a part of $d$, is a neutral ideal, so that $I=B$. Therefore $d$ is large. The conclusion now follows by 8.3.

9. Applications to the coordinatization problem. The following notation was used in the introduction to this paper:

Definition 9.1. If $R$ is a ring, then by $L(R)$ will be meant the set of all principal right ideals of $R$.

As has already been mentioned, if $R$ is a generalized regular ring, then $L(R)$ is a relatively complemented modular lattice. The coordinatization problem is concerned with the converse question of determining whether or not a given relatively complemented modular lattice is coordinatizable in the sense of the next definition.

Definition 9.2. A lattice $B$ is said to be coordinatizable if and only if $B \cong L(R)$ for some generalized regular ring $R$.

This is a modification of the definition CM8.1, where it was required that $R$ be regular. However, if $B$ has a unit, then $R$ must necessarily have an identity, and the results in CM are therefore not affected by this change.

Lemma 9.3. Suppose $M$ is a projective module over a regular ring $R$, and let $R^{\prime}$ be the set of all those endomorphisms of $M$ whose range is finitely generated. Then $R^{\prime}$ is a generalized regular ring and $L(M, R)$ is mapped isomorphically onto $L\left(R^{\prime}\right)$ by the function $G$ such that 


$$
G(N)=\{f \mid f \in L(M, R) \text { and } f(M) \subseteq N\} \quad \text { for all } N \in L(M, R) .
$$

Proof. Clearly $R^{\prime}$ is a subring of the ring of all endomorphisms of $M$, $G(N)$ is a right ideal of $R^{\prime}$ for each $N \in L(M, R)$, and

$$
G\left(N \cap N^{\prime}\right)=G(N) \cap G\left(N^{\prime}\right) \quad \text { for all } N, N^{\prime} \in L(M, R) .
$$

Next observe that the kernel of every member $g$ of $R^{\prime}$ is a direct summand of $M$. In fact, $g(M)$ is a finitely generated submodule of $M$, whence it follows by Kaplansky [8, Theorem 4 and Lemma 4$]$ that $g(M)$ is a direct summand of $M$ and a direct sum of finitely many cyclic submodules $R x_{k}$ each of which is isomorphic to a principal right ideal of $R$. More precisely, there exist idempotent elements $\epsilon_{k}$ such that $\epsilon_{k} x_{k}=x_{k}$ and the mapping $\alpha \rightarrow \alpha x_{k}$ is an isomorphism of $R \epsilon_{k}$ onto $R x_{k}$. For each $x_{k}$ there exists $y_{k} \in M$ with $g\left(y_{k}\right)=x_{k}$, and since $g\left(\epsilon_{k} y_{k}\right)=\epsilon_{k} x_{k}=x_{k}$, it may be assumed that $\epsilon_{k} y_{k}=y_{k}$. Since the compositions of the mappings $\alpha \rightarrow \alpha y_{k} \rightarrow \alpha x_{k}$ is an isomorphism, the latter of these mappings is an isomorphism of $R y_{k}$ onto $R x_{k}$. The sum $N=R y_{0}+R y_{1}+\cdots$ $+R y_{n-1}$ is therefore direct, and $g$ maps $N$ isomorphically onto $g(M)$. From this it readily follows that $M=N+N^{\prime}$ where $N^{\prime}$ is the kernel of $g$.

In the above notation, the isomorphism of $g(M)$ onto $N$ that maps each $x_{k}$ onto the corresponding $y_{k}$ can be extended to an endomorphism $h \in R^{\prime}$ of $M$, and it readily follows that $g h g=g$. This shows that $R^{\prime}$ is a generalized regular ring.

Now consider any $f \in R^{\prime}$. If $g \in R^{\prime}$ and $g(M) \subseteq f(M)$, then by the projectivity of $M$ there exists an endomorphism $h$ of $M$ such that $g=f h$. Since the kernel of $M$ is a direct summand of $M, h$ can be so chosen that $h(x)=0$ whenever $g(x)=0$, and therefore $h \in R^{\prime}$. Consequently $g \in f R^{\prime}$. Thus

$$
G(f(M))=f R^{\prime} \quad \text { for all } f \in R^{\prime}
$$

On the other hand, if $N \in L(M, R)$, then $N$ is a direct summand of $M$, and there exists $f \in R^{\prime}$ with $N=f(M)$, and therefore $G(N)=f R^{\prime}$. This shows that $G$ maps $L(M, R)$ onto $L\left(R^{\prime}\right)$, and also that $G(N)=0$ if and only if $N=0$.

Finally, in order to prove that $G\left(N+N^{\prime}\right)=G(N)+G\left(N^{\prime}\right)$ whenever $N, N^{\prime} \in L(M, R)$, it suffices to consider the case in which $N$ and $N^{\prime}$ are disjoint. Then $M=N+N^{\prime}+M^{\prime}$ for some submodule $M^{\prime}$ of $M$. Let $f$ be the projection of $M$ onto $N$ with kernel $N^{\prime}+M^{\prime}$, and $f^{\prime}$ the projection of $M$ onto $N^{\prime}$ with kernel $N+M^{\prime}$. Then $f f^{\prime}=f^{\prime} f=0$, and $f+f^{\prime}$ is the projection of $M$ onto $N+N^{\prime}$ with kernel $M^{\prime}$. Consequently

$$
\begin{aligned}
G\left(N+N^{\prime}\right) & =G\left(\left(f+f^{\prime}\right)(M)\right)=\left(f+f^{\prime}\right) R^{\prime} \\
& =f R^{\prime}+f^{\prime} R^{\prime}=G(N)+G\left(N^{\prime}\right) .
\end{aligned}
$$

Thus $G$ maps $L(M, R)$ isomorphically onto $L\left(R^{\prime}\right)$.

If the lattice $B$ in 8.3 has a unit, then the representation module $M$ is finitely generated and is therefore projective. An application of 9.3 therefore 
shows that $B$ is in this case coordinatizable. This yields an alternative proof of the Coordinatization Theorem CM8.2. A more general condition that insures the projectivity of $M$ will now be given.

Theorem 9.4. Suppose $B$ is a relatively complemented Arguesian lattice, and assume that there exists a subset $U$ of $B$ such that every finite subset of $U$ is independent and each member of $B$ is contained in the sum of a finite subset of $U$. If, in addition, there exists a large element $d \in B$ such that $B d$ possesses a homogeneous 3-frame, then $B$ is coordinatizable.

Proof. By $8.2, B \cong L(M, R)$ where $R$ is a regular ring and $M$ is a locally projective module over $R$. Let $F$ be the given isomorphism. The conditions imposed on $U$ imply that $M$ is the direct sum of the submodules $F(x)$ with $x \in U$, and since each summand is projective, so is $M$. Hence the conclusion follows by 9.3 .

Observe that if the lattice $B$ is denumerable, then there always exists a set $U$ with the required properties. Thus in particular every simple, denumerable, relatively complemented modular lattice of dimension four or more is coordinatizable.

10. Further results on coordinatization. It is not known whether the limit of a directed system of coordinatizable lattices is coordinatizable. In this section a rather special case of this problem will be treated. To make the formulation of the results more concise the following terminology is introduced:

Definition 10.1. Suppose $B$ is a relatively complemented modular lattice.

(i) By a coordinate system of $B$ is meant an ordered pair $\langle R, F\rangle$ such that $R$ is a generalized regular ring and $F$ is an isomorphism of $B$ onto $L(R)$.

(ii) Two coordinate systems $\langle R, F\rangle$ and $\left\langle R^{\prime}, F^{\prime}\right\rangle$ of $B$ are said to be equivalent if and only if the isomorphism $F^{\prime} F^{-1}$ of $L(R)$ onto $L\left(R^{\prime}\right)$ is induced by an isomorphism of $R$ onto $R^{\prime}$.

(iii) $B$ is said to be uniquely coordinatizable if and only if $B$ is coordinatizable and any two coordinate systems of $B$ are equivalent.

LеммA 10.2. If $R$ is a generalized regular ring and $\epsilon \in R$ is idempotent, then there exists a function $G$ that maps $L(\epsilon R, R)$ isomorphically onto $L(\epsilon R \epsilon)$ in such $a$ way that $G(\alpha R)=\alpha R \epsilon$ for all $\alpha \in \epsilon R$.

Proof. Clearly the given condition defines a monotonic function on $L(\epsilon R, R)$ into $L(\epsilon R \epsilon)$. If $\alpha \in \epsilon R$, then

$$
\alpha R \in R \supseteq \alpha R \alpha R=\alpha R, \quad \alpha R \in R=\alpha R,
$$

because $\alpha=\alpha \gamma \alpha$ for some $\gamma \in R$. Consequently, if $\alpha, \beta \in R$ and $\alpha R \epsilon=\beta R \epsilon$, then right multiplication by $R$ yields $\alpha R=\beta R$. Therefore $G$ is one-to-one. Furthermore, every member of $L(\epsilon R \epsilon)$ is of the form $\alpha R \epsilon$ for some $\alpha \in \epsilon \in \epsilon$, so that $G$ is onto, and is therefore an isomorphism.

THEOREM 10.3 If a relatively complemented modular lattice $B$ is the union of 
an increasing sequence of uniquely coordinatizable principal ideals, then $B$ is coordinatizable.

Proof. Let the given principal ideals be $B a_{n}, n=0,1, \cdots$, and let $\left\langle R_{n}, F_{n}\right\rangle$ be a coordinate system of $B a_{n}$. Then $a_{0}<a_{1}<\cdots$ and, for $n=0,1, \cdots, F_{n+1}\left(a_{n}\right)=\epsilon_{n} R_{n+1}$ where $\epsilon_{n}$ is an idempotent element of $R_{n+1}$. Thus $F_{n+1}$ maps $B a_{n}$ isomorphically onto the principal ideal $L\left(\epsilon_{n} R_{n+1}, R_{n+1}\right)$ of $L\left(R_{n+1}\right)$. By 10.2 there exists an isomorphism $G_{n}$ of $L\left(\epsilon_{n} R_{n+1}, R_{n+1}\right)$ onto $L\left(\epsilon_{n} R_{n+1} \epsilon_{n}\right)$ such that $G_{n}\left(\alpha R_{n+1}\right)=\alpha R_{n+1} \epsilon_{n}$ for all $\alpha \in \epsilon_{n} R_{n+1}$. Consequently $\left\langle\epsilon_{n} R_{n+1} \epsilon_{n}, G_{n} F_{n+1}\right\rangle$ is a coordinate system of $B a_{n}$, and the isomorphism $G_{n} F_{n+1} F_{n}^{-1}$ of $L\left(R_{n}\right)$ onto $L\left(\epsilon_{n} R_{n+1} \epsilon_{n}\right)$ is induced by an isomorphism of $R_{n}$ onto $\epsilon_{n} R_{n+1} \epsilon_{n}$. Without loss in generality it may be assumed that $R_{n}=\epsilon_{n} R_{n+1} \epsilon_{n}$, and that the inducing isomorphism is the identity. This implies that if $x \in B a_{n}$, and if $\alpha \in R_{n}$ is so chosen that $F_{n}(x)=\alpha R_{n}$, then $F_{n+1}(x)=\alpha R_{n+1}$. Thus

$$
F_{n+1}(x)=F_{n}(x) R_{n+1} \quad \text { for all } x \in B a_{n} .
$$

Since $R_{0} \subseteq R_{1} \subseteq \cdots$, the union of the rings $R_{n}$ is a generalized regular ring $R$, and $R_{n}=\epsilon_{n} R \epsilon_{n}$, so that by 10.2 the mapping $I \rightarrow H_{n}(I)=I R$ is an isomorphism of $L\left(R_{n}\right)$ onto $L\left(\epsilon_{n} R, R\right)$. If $x \in B a_{n}$, then

$$
H_{n} F_{n}(x)=F_{n}(x) R \text { and } H_{n+1} F_{n+1}(x)=F_{n+1}(x) R=F_{n}(x) R_{n+1} R \text {. }
$$

Since $R_{n+1} R \subseteq R$ and $F_{n}(x) R_{n+1} \supseteq F_{n}(x)$, it follows that

$$
H_{n+1} F_{n+1}(x)=H_{n} F_{n}(x) \quad \text { for all } x \in B a_{n} .
$$

The isomorphisms $H_{n} F_{n}$ therefore have a common extension $F$ that maps $B$ isomorphically into $L(R)$. Inasmuch as every element of $R$ belongs to one of the right ideals $\epsilon_{n} R$, every principal right ideal of $R$ belongs to one of the lattices $L\left(\epsilon_{n} R, R\right)$. The lattice $L(R)$ is therefore the union of the increasing sequence of principal ideals $L\left(\epsilon_{n} R, R\right)$, whence it follows that $F$ maps $B$ isomorphically onto $L(R)$. Thus $\langle R, F\rangle$ is a coordinate system for $B$.

It is not known whether the hypothesis of the above theorem could be weakened by only requiring the given principal ideal to be coordinatizable. On the other hand, even with the present hypothesis it does not follow that $B$ is uniquely coordinatizable. In fact, the lattice $B$ of all finite dimensional subspaces of an $\boldsymbol{\aleph}_{0}$ dimensional vector space $V$ over a field $F$ always has at least two nonequivalent coordinate systems. For the first coordinate ring $R$ take the ring of all those linear transformations of $V$ into itself whose range if finite dimensional. Considering a fixed basis $\left\{a_{1}, a_{2}, \cdots\right\}$, let the second ring $R^{\prime}$ consist of all those linear transformations that map all but finitely many of the base vectors onto 0 . In each case, associate with each member $N$ of $B$ the right ideal consisting of all those linear transformations in the given ring whose range is contained in $N$. The ring $R^{\prime}$ is the union of an increasing sequence of regular subring $p_{n} R^{\prime} p_{n}$ where $p_{n}$ is the projection that 
maps each of the first $n$ base vectors onto itself and the remaining base vectors onto 0 . On the other hand, if $q_{1}, q_{2}, \cdots$ are projections in $R$, it is easy to show that there exist independent vectors $b_{1}, b_{2}, \ldots$ such that $q_{n}\left(b_{m}\right)=0$ for $m>n$. Consequently there exists a linear transformation $f$ in $R$ such that $f\left(b_{m}\right)=b_{1}$ for $m=1,2, \cdots$, and it follows that $f$ does not belong to any of the subrings $q_{n} R q_{n}$. It follows that $R$ and $R^{\prime}$ are not isomorphic, and that $B$ is therefore not uniquely coordinatizable.

In connection with the last theorem it should be observed that the Isomorphism Theorem CM9.4 does not actually assert that the lattice under consideration is uniquely coordinatizable, for it does not state that the ring isomorphism induces the given lattice isomorphism. It is however easy to show that this is actually the case, and in the present terminology the principal results of $\mathrm{CM}$ can therefore be formulated as follows.

TheOREM 10.4. If a complemented Arguesian lattice $B$ possesses a large partial 3-frame, then $B$ is uniquely coordinatizable.

Proof. The existence of a coordinate system is given in CM8.3, and in CM9.4 it is shown that if $\langle R, f\rangle$ and $\left\langle R^{\prime}, f^{\prime}\right\rangle$ are two coordinate systems for $B$, then $R$ and $R^{\prime}$ are isomorphic. It only remains to show that the ring isomorphism constructed there actually induces the lattice isomorphism $f^{\prime} f^{-1}$. The notation used in the proof of CM9.4 will be employed, and various details from that proof will be freely used. Since in CM the coordinatization was expressed in terms of left ideals, the same will be done here.

An explicit formula for the isomorphism $\bar{h}$ of $R$ onto $R^{\prime}$ was not given, but reference to CM9.3 shows that

$$
\bar{h}(\gamma)=\sum_{i, j<n} \beta_{i} h\left(\alpha_{i} \gamma \beta_{j}\right) \alpha_{j} \quad \text { for all } \gamma \in R .
$$

The desired conclusion, that $\bar{h}$ induces $f^{\prime} f^{-1}$, is equivalent to the assertion that for each $v \in B$ there exists $\gamma \in R$ such that

$$
f(v)=R \gamma \text { and } f^{\prime}(v)=R^{\prime} \bar{h}(\gamma) .
$$

From (1) it follows that

$$
\bar{h}\left(\epsilon_{k}\right)=\epsilon_{k}^{\prime}, \quad \bar{h}\left(\alpha_{k}\right)=\alpha_{k}^{\prime}, \quad \bar{h}\left(\beta_{k}\right)=\beta_{k}^{\prime} .
$$

Consequently (2) holds for $v=b_{k}$ and $\gamma=\epsilon_{k}$, and for $v=c_{k}$ and $\gamma=\eta_{k}$. Furthermore, if $v \in T$ then $v=g(\gamma)$ with $\gamma \in \epsilon_{0} R \epsilon_{0}$, and therefore $v=g^{\prime} h(\gamma)$. Consequently $f(v)=R\left(\gamma-\alpha_{1}\right)$ and $f^{\prime}(v)=R^{\prime}\left(h(\gamma)-\alpha_{1}^{\prime}\right)$, so that (2) holds in this case with $\gamma$ replaced by $\gamma-\alpha_{1}$. The proof will therefore be complete if it is shown that $B$ is generated by the set $T$ together with the elements $b_{k}, c_{k}$.

Let $C$ be the sublattice of $B$ that is generated by the set

$$
T \cup\left\{b_{0}, c_{0}, \cdots, b_{n-1}, c_{n-1}\right\} \text {. }
$$

First consider an element $z \in B$ with $z \leqq b_{0}$. Let $z^{\prime}=\left(z+b_{1}\right) c_{1}$ and choose $z^{\prime \prime} \leqq b_{1}$ 
with $b_{0}+b_{1}=b_{0}+z^{\prime}+z^{\prime \prime}$. Then $z^{\prime}+z^{\prime \prime} \in T$ and $z=b_{0}\left(z^{\prime}+z^{\prime \prime}+b_{1}\right)$, so that $z \in C$. Next consider $z \in B$ with $z \leqq b_{0}+b_{1}$ and $b_{0} z=0$. Choose $z^{\prime} \leqq b_{1}$ with $b_{0}+b_{1}$ $=b_{0} \dot{+} z+z^{\prime}$, and let $z^{\prime \prime}=\left(b_{0}+z^{\prime}\right) c_{1}$. Then $z+z^{\prime}$ and $z+z^{\prime \prime}$ are members of $T$ and $z=\left(z+z^{\prime}\right)\left(z+z^{\prime \prime}\right)$, so that $z \in C$. Since every member $z$ of the ideal $B\left(b_{0}+b_{1}\right)$ can be expressed in the form $z=z b_{0}+z^{\prime}$, it follows that this ideal is contained in $C$. Repeated application of CM1.2 shows that $B\left(b_{0}+b_{1}+\cdots+b_{i}\right)$ $\subseteq C$ for $i=2,3, \cdots, n-1$, and the case $i=n-1$ yields the desired conclusion that $B=C$.

\section{BIBLIOGRAPHY}

1. E. Artin, Coordinates in affine geometry, Rep. Math. Coll., Notre Dame 1940, pp. 15-20.

2. R. Baer, Linear algebra and projective geometry, New York, 1940.

3. O. Frink, Complemented modular lattices and projective spaces of infinite dimension, Trans. Amer. Math. Soc. 60 (1946), 452-467.

4. K. D. Fryer and I. Halperin, The von Neumann coordinatization theorem for complemented modular lattices, Acta Szeged 17 (1956), 203-249.

5. B. J6nsson, Extensions of von Neumann's coordinatization theorem, Lattice theory, Proc. Sympos. Pure Math. Vol. 2, pp. 65-70, Amer. Math. Soc., Providence, R. I., 1961.

6. - Modular lattices and Desargues theorem, Math. Scand. 2 (1954), 295-314.

(1960), 64-94.

8. I. Kaplansky, Projective modules, Ann. of Math. 68 (1958), 372-377.

9. J. von Neumann, Continuous geometry, Princeton Univ. Press, Princeton, N. J., 1960.

UNIVERSITY OF MINNESOTA,

MinNeAPOLIS, MinNesota 